\title{
RECONCILIACIÓN DE MALÍ; MAYO EN KIDAL, INFLEXIÓN EN LA SEGUNDA FASE DEL PROCESO:
}

\author{
Juan Carlos Castilla ${ }^{1}$ \\ EUTM, Mali
}

\begin{abstract}
Resumen:
La reconciliación es uno de los retos de la consolidación de la paz. El ejercicio de la soberanía por parte de las instituciones locales, puede originar diferencias con las organizaciones internacionales durante la transformación del conflicto. El desafío es coordinar estrategias y medidas en el tiempo. El proceso de reconciliación comenzó en Malí con un gobierno de transición. Durante el año 2014, el gobierno ha tratado de impulsar el proceso, en un contexto en el que se han reproducido los enfrentamientos tribales, se han dado nuevos choques entre el MNLA y las Fuerzas Armadas malíes, y dónde sigue influyendo la actividad terrorista yihadista. IBK se refirió a aspectos fundamentales para el futuro inmediato del proceso tras los acontecimientos de mayo en Kidal. Tras estos acontecimientos, las negociaciones de paz se desarrollan con la mediación argelina. La complementariedad entre la justicia nacional y la internacional será una de las claves, lo que a su vez puede interrelacionar con la potencial re-integración futura de excombatientes.
\end{abstract}

Palabras clave: Sahel, Malí, Diálogo, Verdad, Justicia, Reconciliación, Corte Penal Internacional, MINUSMA, EUCAP Sahel Mali, EUTM Mali, SSR, DDR.

Title in English: "Reconciliation in Mali; May in Kidal, Watershed in the Process's Second Phase”

\begin{abstract}
:
Reconciliation is one of the challenges within the peace consolidation. The exercise of sovereignty by the local institutions may lead to differences with international organizations during the conflict transformation. Another challenge is to coordinate strategies and actions at the time. In Mali, the reconciliation process began with a transitional Government. During 2014, the Government have tried to boost the process in a context in which the tribal clashes reproduced, new fighting between the MNLA and Malian armed forces were on the spotlight, and the jihadist terrorist activity continues their intend to influence. IBK addressed key issues for the future of the process immediately after the May events in Kidal. Following these events, the peace negotiations develop with Algerian mediation. The complementarity between national and international justice is a key that can be linked with the future potential re-integration of ex-combatants.
\end{abstract}

Keywords: Sahel, Mali, Dialogue, Truth, Justice, Reconciliation, International Criminal Court ICC, MINUSMA, EUCAP Sahel Mali, EUTM Mali, SSR, DDR.

\section{Copyright $@$ C UNISCI, 2015.}

Las opiniones expresadas en estos artículos son propias de sus autores, y no reflejan necesariamente la opinión de UNISCI. The views expressed in these articles are those of the authors, and do not necessarily reflect the views of UNISCI.

\footnotetext{
${ }^{1}$ Juan Carlos Castilla Barea es Teniente Coronel de Artillería del EM y miembro de la misión de paz, EUTM, en Mali (Bruselas).

E-Mail: j.c.castilla@hotmail.com.
} 


\section{Introducción a las causas y a la gestión internacional del conflicto en Malí}

"Tal como nuestra experiencia en Sudáfrica nos ha enseñado, cada sociedad debe descubrir su propia ruta a la reconciliación. La reconciliación no puede ser impuesta desde fuera, nadie más puede guiarnos a nuestro destino: debe ser nuestra propia solución. Esto implica un largo y doloroso viaje, afrontando el dolor y el sufrimiento de las víctimas, entendiendo la motivación de los perpetradores, juntando a comunidades enfrentadas, tratando de encontrar un camino para la justicia, la verdad, y en último término, la paz. [...] nuevas soluciones se deben idear que sean apropiadas al contexto particular, historia, y cultura en cuestión."

Desmond Tutú, 2003. ${ }^{2}$

En Malí se desarrolla un conflicto socio-internacional de carácter etno-político prolongado. Sus causas primigenias se remontan al menos a tiempos de la descolonización francesa. En este conflicto se dan varias crisis en su discurrir histórico, en forma de rebeliones armadas (1962-1964, 1990-1996, 2006-2009, 2011-2013). Argelia tiene un papel histórico destacado en la gestión internacional de las crisis desde los años 90; actúa como mediador entre los tuaregs y el gobierno de Bamako, y tiene un rol relevante en la definición y firma de los acuerdos de paz correspondientes. ${ }^{3}$ La mediación de Argelia ha comenzado de forma abierta tras los acontecimientos de mayo de 2014 en Kidal.

Además de las distintas rebeliones tuaregs, la militarización de la vida política en Malí también es causa coadyuvante de la conflictividad. Se dieron varios golpes de estado en Bamako, desde su independencia hasta la última crisis. ${ }^{4}$ Dentro del contexto del histórico conflicto, también tuvieron su importancia las emergencias alimentarias causadas por periodos de sequías (1969-1974 y 1982-1984). Lo anterior agravaba la debilidad económica estructural, lo que unido a las represiones que seguían a las rebeliones, causó éxodos masivos de población, también alentados por los incentivos económicos que ofrecía Libia.

Este conflicto se desarrolla en un país con un Estado vulnerable, en el que grupos armados usurparon la autoridad del Estado. Entre 2011 y 2013 se evidenció la debilidad estatal, incluso más de lo que cabía esperar con antelación, hasta casi el colapso. A finales de 2011, una nueva crisis se desencadenaba en Malí, entre otros factores, por la vuelta de combatientes tuaregs, procedentes de Libia. El régimen libio cayó a raíz de una operación militar de la OTAN; operación en la que Francia tuvo un papel destacado. ${ }^{5}$

Aconteció una doble crisis humanitaria, alimenticia en la generalidad del país, y más acuciante entre los refugiados, de dentro y fuera del país, que aún continúa. Tras la reconquista del norte a partir de enero de 2013, las cifras de ACNUR estimaban que 430.000 malienses tuvieron que dejar sus casas desde el inicio de la crisis, 280.000 desplazados internos y 177.000 refugiados en otros países, principalmente en Mauritania (74.024), Níger (50.000) y Burkina Faso (49.975). ${ }^{6}$

\footnotetext{
2 Bloomfield, D., Barnes, T., \& Huyse, L. (2003): Reconciliation after violent conflict. A handbook, Halmstad, Sweden: Institute for Democracy and Electoral Assistance IDEA. Prefacio.(Traducción propia).

3 Acuerdo de Tamanrasset (Argelia) de enero 1991 y el Pacto Nacional de 1992, hasta llegar al Acuerdo de Argel de 1996, Acuerdo de Argelia de 2006.

4 En 1968, el golpe del teniente Moussa Traoré. En marzo de 1991, se produjo el golpe del teniente coronel paracaidista Amadou Toumani Touré (ATT). En marzo de 2012, el golpe del Capitán Sanogo.

5 Se puede considerar a la acción de la OTAN como un detonante indirecto y coyuntural que facilitó el estallido de la última rebelión tuareg en el norte de Malí; en parte fue un 'efecto colateral' no deseado.

6 "Llevando esperanza a los refugiados en Malí", en http://acnur.es/crisis-en-mali.

7 Se han dado pasos para mitigar la emergencia alimentaria; pero en el aspecto de los refugiados, la crisis solo se puede considerar solucionada con el retorno final de éstos. Este aspecto presenta una dificultad especial en gran parte de los conflictos, y se relaciona con los procesos de reconciliación y reintegración.
} 
Esta afectación a países limítrofes suponía uno de los supuestos de intervención de la Comunidad Económica de Estados de África Occidental (CEDEAO) en conflictos internos de países miembros; sin embargo, las cláusulas de asistencia mutua no operaron en $2012 .{ }^{8} \mathrm{La}$ crisis desencadenada era una claramente "nacional con implicaciones internacionales"9

Sin duda, la naturaleza principal del cambio brusco operó a nivel político. Se trataba de una crisis de seguridad. Desde Max Weber se admite que la seguridad de los ciudadanos es un servicio esencial a dar por un Estado, que detenta la única forma legítima del uso de la violencia. Por lo anterior, la seguridad tiene origen en las instituciones políticas; a menos que las autoridades del Estado dejen de ejercer un control efectivo sobre el territorio y la población, y otros actores asuman este rol.

En Malí, a lo político-social de índole étnica, se unió la confluencia del crimen organizado con el terrorismo yihadista global, ambos presentes en Malí.

Tras el golpe de marzo de 2012, se instauró un Gobierno de transición en Malí; no contaba inicialmente con el reconocimiento de la CEDEAO, de la Unión Africana (UA), ni del resto de la comunidad internacional. La aproximación de la comunidad internacional hacia Bamako empezó a cambiar, tras la declaración de independencia de Azawad en abril, y a medida que los yihadistas se hacían con el control en el norte en junio de 2012; la amenaza potencial ya no se limitaba a Malí. Se constituía un santuario para la yihad global. Ésta es enemiga declarada de las organizaciones internacionales como la Unión Africana (UA), la Unión Europea (UE) y las Naciones Unidas (ONU). Tras la reacción internacional de enero de 2013 se restauró la integridad territorial de Malí, dónde aún hoy persiste la amenaza terrorista. El terrorismo no es la causa principal del conflicto, aunque sí es un factor relevante en el desarrollo de la última crisis.

En enero de 2013, en respuesta a la ofensiva yihadista hacia el sur, Francia lanzó la Operación Serval recuperándose en poco tiempo la integridad territorial. Fue una operación con una legitimidad inicial limitada; se desencadenó a petición del Gobierno de transición, que carecía aún de un suficiente respaldo internacional. La operación se consiguió legitimar en poco tiempo, con la aprobación unánime de las organizaciones regionales y de la ONU.

Tras la decisiva intervención francesa, tropas de países de la CEDEAO se integraron en la Misión Internacional de Apoyo a Malí con Liderazgo Africano (AFISMA en inglés). En marzo de 2013, la operación Serval, con el apoyo de las FAS malíes y de AFISMA, había conseguido prácticamente recuperar el control del norte de Malí.

El 27 de marzo de 2013, los Jefes de Estado Mayor de los países de la CEDEAO solicitaban la transformación de AFISMA en una misión de ONU. ${ }^{10}$ Un día antes, el Secretario General anunciaba la misión de la ONU en su informe sobre Malí.

En el informe de situación sobre Malí de 26 de marzo de 2013, el Secretario General de las Naciones Unidas, Ban Ki-moon, recogía la opinión del Gobierno de transición de Malí sobre la situación política en los términos que siguen: “...las causas básicas del conflicto, a saber, la mala gobernanza, la corrupción y un estado de derecho y sector de la seguridad

8 Desde 1975, la CEDEAO intenta promover la integración económica y garantizar la seguridad regional. En este ámbito son significativos el Protocolo de No Agresión de 1978 y el Acuerdo de Asistencia y Defensa Mutua de 1981; el segundo incluía un procedimiento de respuesta colectiva aplicable a conflictos externos e internos. En estos últimos casos, la intervención se justificaba en base a su impacto exterior, por ser una amenaza seria a la paz y a la seguridad regional (Díez Alcalde, febrero 2013).

9 Véase, De Castro Sánchez, C. \& Quesada Alcalá, C. (2007): "El derecho internacional de la gestión de crisis", en IUGM: Realidades y Perspectivas de la Gestión Internacional de Crisis, p. 61.

10 Comunicado de prensa de la CEDEAO del 27MAR13, disponible en

http://news.ecowas.int/presseshow.php?nb=082\&lang=en\&annee=2013. 
ineficaces, tendría que abordarlas el gobierno elegido" (elecciones de julio de 2013). ${ }^{11}$ En el mismo párrafo continuaba el informe: "Algunos interlocutores expresaron su preocupación por que la continua injerencia de elementos implicados en el golpe del 21 de marzo de 2012 debilitaba las instituciones. Confirmaron que existían importantes divisiones entre los agentes políticos y dentro del ejército..."

El 25 de abril de 2013 se aprobaba la Misión Multidimensional e Integrada de las Naciones Unidas para la Estabilización en Malí (MINUSMA), con arreglo a lo dispuesto en el Capítulo VII, planeaba contar hasta con 11.200 soldados y 1.440 policías. Se autorizaba a las tropas francesas, a operar en apoyo a MINUSMA, como una fuerza paralela. ${ }^{12} \mathrm{La}$ transferencia de autoridad desde AFISMA a MINUSMA fue el 01 de julio de 2013. ${ }^{13}$

En la segunda quincena de abril, y tras una remodelación del Gobierno de Bamako, se produjo una escalada verbal en medios locales e internacionales con respecto a las relaciones entre las autoridades locales y MINUSMA. Entre los aspectos debatibles se encontraba el reparto de tareas en relación con las elecciones comunales a celebrar en el último trimestre de 2014, en particular sobre qué fuerzas habían de encargarse de prestar seguridad.

En abril, el Jefe de la MINUSMA informaba que sus efectivos eran cercanos al sesenta por ciento y de la urgente necesidad de completar su despliegue. ${ }^{14}$ MINUSMA se centra en apoyar la estabilización, el proceso político y los esfuerzos de reconciliación, así como en la protección de los civiles y de los derechos humanos, por lo que necesita de efectivos en los ámbitos de la seguridad militar y policial, así como expertos en áreas civiles. ${ }^{15}$ El ámbito judicial es también relevante.

Con ocasión de una visita oficial del Primer Ministro malí a la ciudad de Kidal, el 17 de mayo de 2014, se produjeron graves enfrentamientos entre grupos armados tuaregs, que mantenían de facto el control de la ciudad, y las Fuerzas Armadas malíes a las que volvieron a imponerse. ${ }^{16} \mathrm{El}$ cese el fuego se establecía el 23 de mayo. Estos hechos desencadenaron una escalada verbal por parte de las autoridades locales y abría un período de incertidumbre para el proceso de reconciliación. Inmediatamente, tras los episodios en Kidal, el Ministro de Defensa francés viajaba a Argelia. ${ }^{17}$ Poco después, tras una nueva resolución del CSNU (S/RES/2164 (2014)) el 25 de junio, ${ }^{18}$ y una entrevista con Ban Ki Moon, ${ }^{19}$ el Presidente

11 Énfasis en cursiva del autor. "Informe de Situación en Malí", UN, en

http://www.un.org/en/ga/search/view doc.asp?symbol=S/2013/189.

12 Resolución 2100, párrafo 18.

13 MINUSMA constituyó, entre otros aspectos, una alternativa de financiación viable a AFISMA. Dado el origen de la mayoría de las tropas, adolece también desde el principio de carencias operativas significativas. La fuerza paralela francesa es capaz de reaccionar ante situaciones graves en ciudades clave como Kidal.

14 "Window of Opportunity' for Peace in Mali Remains Open, but Requires Sustained Commitment, Senior Official Tells Security Council", UN, en http://www.un.org/News/Press/docs/2014/sc11360.doc.htm 4.

15 "La comunidad internacional debe seguir apoyando la recuperación de Mali", UN (enero 2014), en http://www.un.org/spanish/News/story.asp?NewsID=28496\&Kw1=minusma\#.UuybZP15N5I.

16 En estos acontecimientos se evidenció de nuevo la debilidad de las Fuerzas Armadas malíes frente a los grupos armados presentes en Kidal, en un complicado contexto de seguridad, en el que las fuerzas de MINUSMA y francesas tratan de evitar que el proceso descarrile de nuevo.

17 Anteriormente la diplomacia malí había conseguido estrechar lazos con Marruecos. Esto es relevante, ya que la actualidad del proceso de paz evidencia de nuevo que también son necesarias las relaciones con Argelia para la solución del problema en el norte malí y en especial en Kidal. A este respecto, es significativo que Radio France Internacional tras informar sobre los enfrentamientos en la ciudad ocurridos el 17 de mayo de 2014, y el posterior cese el fuego, ponga el foco en la visita del ministro de defensa Le Drian a Alger, especialmente en relación con la lucha contraterrorista en el norte de Malí. Véase, http://www.rfi.fr/afrique/20140520-mali-cessezle-feu-respecte-otages-liberes-kidal-mnla/\#./?\& suid=140058637927403696811892032933.

18 En la resolución 2164 se extiende el mandato de MINUSMA hasta el 30 de junio de 2015.

19 "Readout of the Secretary-General's meeting with H. E. Mr. Ibrahim Boubacar Keita, President of Mali", UN (junio 2014), en http://www.un.org/sg/offthecuff/index.asp?nid=3460. 
Ibrahim Boubacar Keita (IBK) se avenía a mantener negociaciones en Argelia. ${ }^{20}$ Tras un intercambio de prisioneros, la primera ronda de negociaciones se mantenía entre el 16 y el 24 de julio. Se conseguía establecer una hoja de ruta, que incluía una segunda ronda de diálogo (prevista inicialmente para mediados de agosto), con la finalidad de llegar a un acuerdo de paz global, tal como se estipulaba en el acuerdo preliminar de Uagadugú de junio de 2013.

La resolución 2164 volvía a elogiar los esfuerzos en el ámbito institucional maliense, la reversión de la crisis volviendo a la normalidad constitucional, proceso que culminó con las elecciones presidenciales de 28 de julio y 11 de agosto, y legislativas de 24 de noviembre y 15 de diciembre de 2013. Entre otras reformas, también merece mención la refundación de algunas instituciones para reforzar el Estado, entre éstas las Fuerzas Armadas y los Cuerpos de Seguridad. ${ }^{21}$ Una integración deficiente de los tuaregs en el ámbito político, y periodos de seguridad deficiente afectados por varias rebeliones ${ }^{22}$, son las causas principales de los picos de fragilidad $^{23}$ en la historia reciente de Malí, junto a una estructural debilidad económica. A la problemática tampoco son ajenos los factores sociales.

En el corto y medio plazo, la efectividad de la seguridad en Malí seguirá dependiendo de los esfuerzos de organizaciones internacionales en conjunción con esfuerzos de países singularmente interesados en el país. ${ }^{24}$ Organizaciones como la UE y la ONU pueden jugar un papel clave en el aumento de la legitimidad de la seguridad. Un entorno controlado por la seguridad internacional, y la ayuda al desarrollo de organizaciones como la $\mathrm{UE}^{25}$, puede permitir a Malí recuperar su estabilidad, lo que a su vez puede permitirle ir saliendo de la fragilidad extrema.

Junto a estos sujetos jurídicos del derecho internacional (estados y organizaciones internacionales) también intervienen, tanto en la gestión de la crisis como en el proceso de transformación del conflicto, otras organizaciones no gubernamentales y actores de la sociedad civil. La coordinación entre un tipo y otro de actores es conveniente. La coordinación de aspectos civiles y militares dentro de la variedad de instrumentos de los que disponen las organizaciones internacionales para la gestión de crisis es capital.

La reconciliación se encuentra entre los grandes retos de la consolidación de la paz. El proceso en Malí requiere el esclarecimiento de la verdad, y debe suponer la rendición de cuentas de aquellos que han cometido crímenes.

Se sostiene la tesis de que hasta ahora, se distinguen dos fases en el proceso de reconciliación malí, marcadas por la vuelta a la normalidad constitucional tras los procesos electorales que culminan a finales de 2013. La primera corresponde al gobierno de transición y primeros meses del primer gobierno de IBK, el proceso se configuraba en base a una comisión nacional tendente al esclarecimiento de la verdad, en conjunción con la apelación a la Corte Penal Internacional (CPI), para la investigación de los crímenes desde principios de 2012 con ocasión de la última crisis. La segunda comienza a materializarse con la sustitución de la Comisión del Diálogo y la Reconciliación por la Comisión por la Verdad, la Justicia y la

\footnotetext{
20 Entrevista en http://www.rfi.fr/emission/20140627-ibrahim-boubacar-keita-president-republique-malienne/.

$21 \mathrm{El}$ sector de justicia en Malí presenta asimismo grandes oportunidades de mejora.

$22 \mathrm{La}$ legitimidad del estado, en su intento de garantizar la seguridad se ha deteriorado en ocasiones, por episodios de represión excesiva contra la población.

23 Desde su independencia en 1960, han sufrido hasta cuatro rebeliones en el norte del país.

24 En este párrafo 'seguridad' se refiere más a su significado tradicional, que a acepciones más modernas como el de 'seguridad humana'.

25 Ver Informe Europeo sobre el Desarrollo: "Superar la fragilidad de África. Forjar un nuevo enfoque europeo", (2009), en

http://ec.europa.eu/europeaid/what/development-policies/research-

development/documents/erd_report_2009_es.pdf.
} 
Reconciliación a principios de 2014. En esta segunda fase, los sucesos de mayo en Kidal suponen un punto de inflexión en las negociaciones políticas, tras el cual, la mediación argelina es de nuevo un aspecto visible y relevante del proceso. El ejercicio de la soberanía en coordinación con la asistencia internacional serán claves en la evolución del proceso, y en particular, lo relativo a la complementariedad entre instancias judiciales nacionales y la CPI.

Este documento se desarrolla en tres bloques. En el primero se introducen conceptualmente los términos 'proceso de reconciliación', se hace de forma principalmente genérica. Seguidamente se explican los hitos fundamentales de las fases del proceso identificadas en Malí. En el tercero se analiza el caso ante la CPI, en aras a dilucidar algunas claves técnico-jurídicas que nos permiten comprender la flexibilidad implícita en la llamada complementariedad entre instancias judiciales nacionales y la Corte. Finalmente se presentan las conclusiones.

\section{Aproximación conceptual a la reconciliación en su contexto}

En esta aproximación se abordan cuestiones relativas a la definición del proceso de reconciliación como tal. Se incide en que la propiedad del mismo es local, y tienen especial cuota de propiedad quienes ejercen la soberanía de forma legítima. Se finaliza presentando una taxonomía sobre los tipos de procesos, en virtud de la configuración de comisiones e instancias judiciales que pueden ser parte del mismo.

\section{La reconciliación y la gestión de crisis}

David Bloomfield, experto en mediación, considera que la reconciliación y la construcción de la democracia pueden avanzar de forma interrelacionada. El proceso de la reconciliación implica el largo plazo, afecta tanto a las víctimas como a los causantes del dolor, en gran parte de los casos requiere llegar al esclarecimiento de la verdad sobre el pasado para afrontar el futuro, y normalmente llegar a una forma de justicia, retributiva o restaurativa. La reconciliación puede verse pospuesta en aras a completar otras prioridades y urgencias, como el restablecimiento de un régimen democrático, ${ }^{26}$ esto último es de especial aplicación al caso de Malí.

Por otra parte, Luc Huyse desarrolla el concepto de la reconciliación como proceso a partir de preguntas básicas sobre lo qué es y a quiénes afecta, ya introducidas por el coeditor antes citado, y además sobre el cómo y el cuándo. ${ }^{27}$ Enfatiza que, a priori no se puede considerar un proceso lineal; no obstante, típicamente cabe afrontar ciertas fases.

La primera consistente en reemplazar el temor por la coexistencia no violenta, para lo cual, es necesario conseguir un entorno suficientemente seguro, ${ }^{28}$ un mínimo de seguridad física, sin el cual el camino a la reconciliación no es transitable.

Cuando ya el miedo no rige, es tiempo de construir confianza entre víctimas y agresores, y como subproducto, ser capaz de individualizar las culpas frente a las generalizaciones comunitarias, desagregar al individuo de la colectividad; para acometer esto es necesario un mínimo de instituciones en funcionamiento.

La tercera fase acerca a las partes hacia la empatía, es el momento de las comisiones de la verdad, ya que ésta es una precondición para la reconciliación, concurrentemente es tiempo

26 Véase, Bloomfield, Barnes \& Huyse, op. cit., pp 10-18.

27 Ibid., pp. 11-32.

28 Es frecuente encontrar en misiones militares el término SASE: Safe And Secure Environment. 
de encontrar intereses comunes de futuro. Acompañando a todas las fases se han de introducir los códigos de conducta democráticos, y conceptos justos sobre el orden socio-económico en las comunidades a reconciliar.

Huyse postula que tras un conflicto violento, para ir consiguiendo la coexistencia pacífica, la confianza, la empatía y la cultura democrática, se necesitan ciertos instrumentos. Se trata de curar ('healing'), no solo a los individuos sino a las comunidades (curación orientada a la normalización de la vida comunitaria), de esclarecer la verdad histórica escuchando a todas las partes, de hacer justicia de alguna forma (retributiva o restaurativa), y de reparar a las víctimas (material y psicológicamente si es posible). ${ }^{29}$ La reconciliación es esencial para la prevención de nuevos enfrentamientos armados dentro del mismo conflicto. Huyse, al igual que Bloomfield, indica que una de las grandes dificultades a la hora de definir la estrategia para la reconciliación es la secuenciación, el cuándo, el orden de acciones y cuán rápidas han de ser, es decir, el 'timing' y el 'tempo'.

En el siguiente cuadro se identifican los procesos típicos de la gestión de crisis y transformación de conflictos. Entre otros se incluyen tres procesos tipo que conforman la hoja de ruta político social para sociedades en transición, como en el caso de estudio. Se trata de la observación de elecciones, los procesos de Desarme, Desmovilización y Re-integración (DDR), y los procesos de reconciliación. Asimismo se destacan en la figura, las reformas en el sector de la seguridad y del sector judicial, ya que estos, entre otros, son procesos que se relacionan directamente con la reconciliación en sus primeras fases y tienen una gran influencia en el largo plazo.

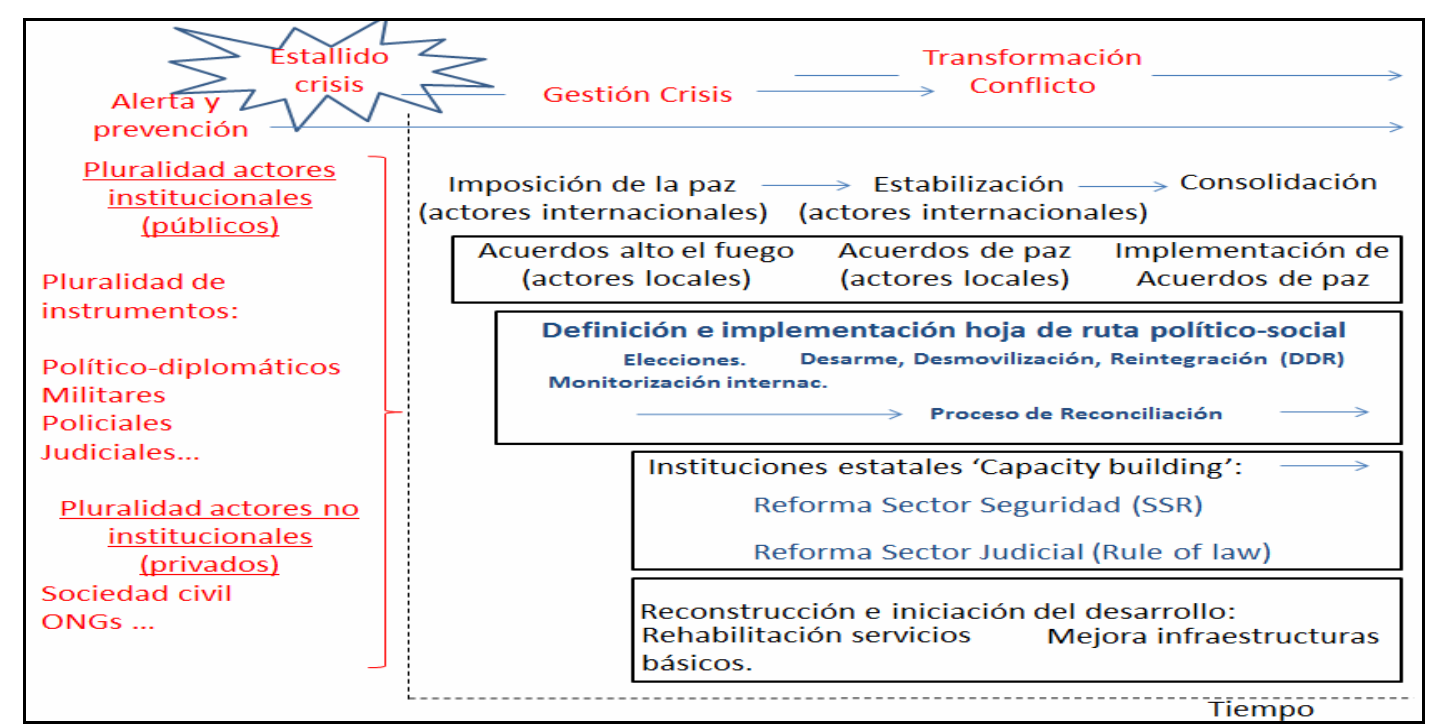

Figura 1. Identificación de procesos típicos. Énfasis en la Reconciliación y Reintegración. (Elaboración propia)

En confluencia con la figura anterior, se muestra a continuación el enfoque temporal que la Comisión Europea presenta como aproximación a la transformación del conflicto. ${ }^{30} \mathrm{Se}$ resaltan igualmente aquellos elementos más relacionados con la reconciliación.

29 La confluencia de instrumentos de Huyse, según el autor, es una adaptación del trabajo de Lederach.

$30 \mathrm{Al}$ igual que en la obra de Huyse, también supone una adaptación del trabajo de Lederach. 


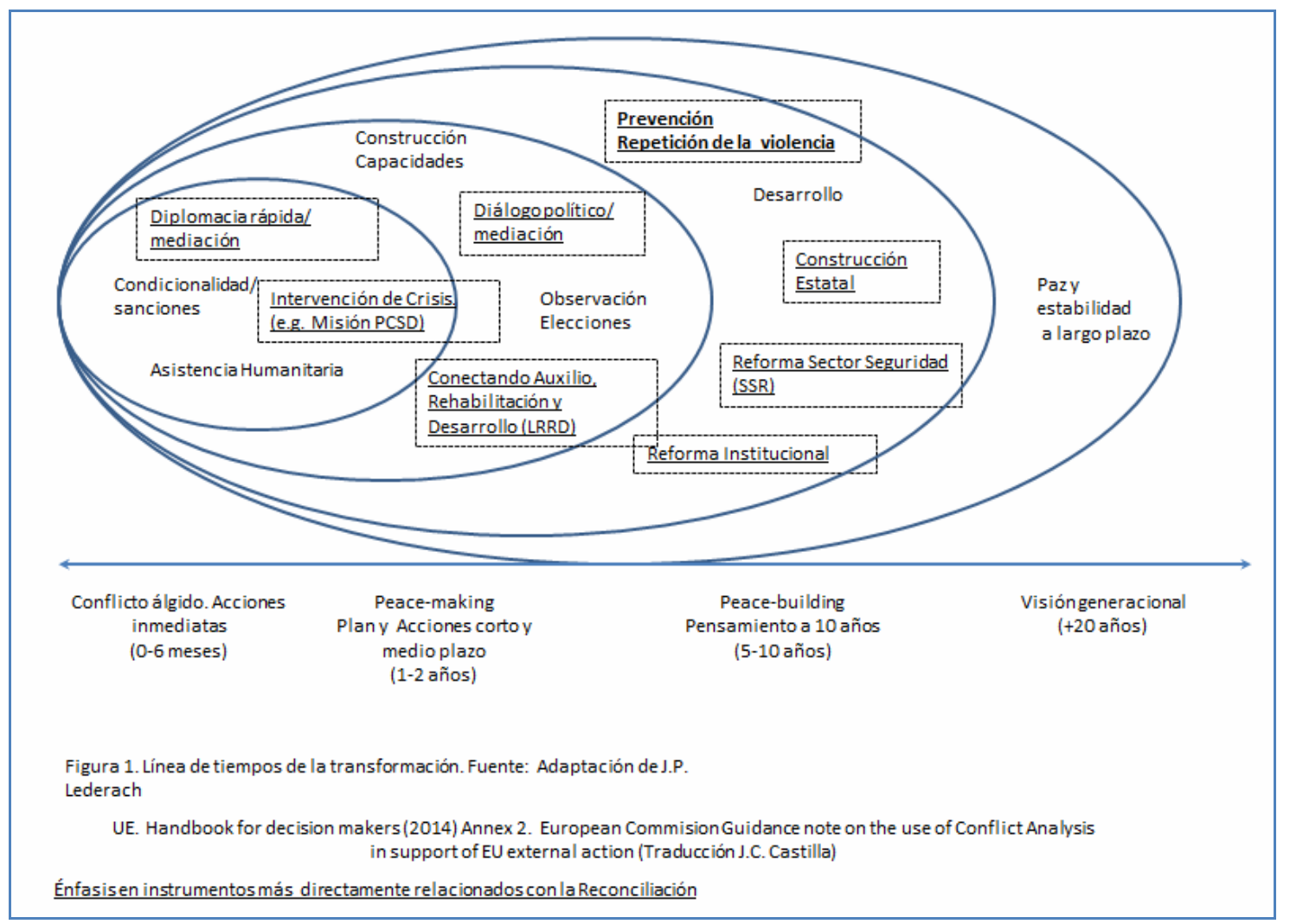

Figura 2. Transformación del conflicto según la Comisión Europea, énfasis propio relativo a la reconciliación. ${ }^{31}$

Por otro lado, la reintegración de antiguos combatientes es la posible culminación de otro proceso, que normalmente viene precedido del acantonamiento de las facciones armadas, su desarme, y desmovilización, lo que se conoce como proceso DDR. Tal como señala Huyse, parece obvio que, según el 'timing' dentro del plan de acción hacia la reconciliación, la justicia retributiva puede hacer más dura la reintegración. "El lugar de la justicia en general, y de los juicios en particular, en la agenda post-conflicto depende de la particular conjunción de fuerzas políticas, culturales e históricas." 33

\section{El control nacional}

Un aspecto del arte de la gestión de crisis, entendida en sentido temporal amplio, es la secuenciación correcta en la implementación de dichos procesos. Es un lugar común que la resolución o transformación del conflicto ha de ser propiedad principal de las partes afectadas directamente. La Resolución 1645, por la que se crea la Comisión de Consolidación de la Paz de las NNUU, concede gran importancia al papel de las instituciones locales, al tratar de asegurar el llamado "control nacional". ${ }^{34}$ Sin duda, entre las partes están las instituciones

31 Ministry of Defence and Sports of the Republic of Austria. (2014). A handbook for decision makers. The Common Security and Defence Policy of the European Union. Viena: Armed Forces Printing Centre. Annex 2. 32 Véase, Bloomfield, Barnes \& Huyse, op. cit., p. 106.

33 Ibid., p. 103.

34 “Afirmando que, cuando se hayan establecido, los gobiernos y autoridades nacionales y de transición de los países que salen de un conflicto o que corren el peligro de que se reanude tienen la responsabilidad primordial de determinar sus prioridades y estrategias de consolidación de la paz después de los conflictos, con el fin de asegurar el control nacional". Resolución disponible en http://www.un.org/es/comun/docs/?symbol=S/RES/1645\%20(2005)\&lang=s. 
del Estado en las que ocurrió el enfrentamiento. Una vez que estas instituciones cuentan o recuperan la necesaria legitimidad, deben ser el actor político clave para el diseño y la puesta en práctica de las distintas medidas en ejercicio de su soberanía; y, con su consentimiento, pueden ser asistidas por las organizaciones internacionales. ${ }^{36}$

El ejercicio de la soberanía por parte de las instituciones locales, puede originar diferencias con las organizaciones internacionales, ${ }^{37}$ durante la transformación del conflicto. El desafío es coordinar estrategias y medidas en el tiempo. Así por ejemplo, la Unión Europea lanzó una misión civil de gestión de crisis con vocación regional, EUCAP Sahel Níger. A ésta, siguió una misión de asistencia a las reformas del sector de seguridad militar en Malí, EUTM Mali, ${ }^{38}{ }^{39}$ y se vio avocada ${ }^{40}$ a aprobar una nueva misión local de 'capacity building', EUCAP Sahel Mali, centrada en las reformas policiales de cuerpos de seguridad civiles y militares, ${ }^{41}{ }^{42}$ en lugar de escalar y re-denominar la original que venía funcionando en el país vecino. La flexibilidad europea supone, en cierta forma, una concesión de su vocación constructivista regional a la complementariedad con las relaciones bilaterales.

Hay que tener en cuenta que en Malí, durante la definición de la hoja de ruta en 2012 y 2013, se contaba con un gobierno de transición, que contaba con una legitimidad limitada, cuyo principal sustento era el apoyo internacional; a lo que contribuía sin duda la amenaza terrorista salafista que se proyectaba más allá de Malí. ${ }^{43}$ Tras las elecciones presidenciales y legislativas, la posición del gobierno, avalado por los votos, es más legítima ${ }^{44}$ para poder

35 Si la población local percibe que no son los propietarios del proceso, la gestión internacional puede fracasar, tal como indican el Cor. (ET) D. Pablo Resa Ancín, y D. Luís Antonio Peral Fernández, investigador del Ramón y Cajal del centro de estudios políticos y constitucionales. "Actuación de las fuerzas armadas en la consolidación de la paz”. CESEDEN. 2006. La posibilidad de que los gobiernos nacionales soliciten el fin de la colaboración (Res 1645, artículo 22) de la Comisión como terminación de la relación ahonda en la idea de la propiedad del proceso por parte de las autoridades locales.

36 Es importante señalar que la reconciliación y la reinserción son procesos principalmente políticos, y por tanto, sujetos a la voluntad soberana de los gobernantes que legítimamente marcan el rumbo de sus poblaciones.

37 Véase Barbé, E. (2012): Cambio mundial y gobernanza global: la interacción entre la Unión Europea y las instituciones internacionales, Barcelona, Tecnos SA. En lo relativo a la aspiración a la 'gobernanza global', se identifica que la aproximación de la UE implica un constructivismo institucional impregnado de espíritu reformista hacia las instituciones internacionales, que colisiona con una revitalización de una corriente soberanista, apoyada por algunas potencias emergentes; este reformismo europeo se llega a percibir por ciertos países como un intento de neocolonialismo. Lo anterior enmarca la interacción de la UE con las instituciones internacionales.

38 Castilla, J.C. (2013): "La reforma de las Fuerzas Armadas de Mal y el futuro de EUTM", Instituto Español de Estudios Estratégicos (IEEE), en www.ieee.es.

39 Desde el 24 de octubre de 2014, un general español manda la misión EUTM Malí. Véase, http://www.defensa.gob.es/gabinete/notasPrensa/2014/10/DGC-141024-Toma-de-posesion-EUTM-Mali.html.

40 Casi desde el inicio de la fase inicial del despliegue de EUTM Mali, el Consejo examinaba las opciones de desplegar una misión civil. "El 27 de mayo de 2013, el Consejo reiteró su disposición a examinar, en particular en el marco de la PCSD, las opciones con vistas al apoyo urgente a las autoridades malienses en el ámbito de la seguridad interior y la justicia, incluida la lucha contra el terrorismo y la delincuencia organizada." (Preliminar (5)) decisión no 2014/219/PESC, en http://www.boe.es/doue/2014/113/L00021-00026.pdf.

41 Esta misión fue aprobada el 15 de abril de 2014. Su Jefe de Misión fue nombrado el 26 de mayo. Los órganos de planeamiento en el Servicio Exterior de la UE tienen una capacidad limitada en virtud de sus recursos humanos (La CPCC alrededor de unas 60 personas), y entre abril y mayo la atención y las actividades de planeamiento, Concepto de Gestión de Crisis (CMC), se centraban en la crisis de Ucrania.

42 Son todas misiones de construcción de capacidades institucionales, misiones de 'capacity building', sus objetivos se encuadran en el sector de 'Security Sector Reform' (SSR).

43 Castilla, J. C. "Evolución estratégica y operacional del terrorismo en África: el caso de Malí", Instituto Español de Estudios Estratégicos (IEEE), en www.ieee.es.

44 Todos los sujetos de derecho internacional, Estados u organizaciones internacionales, sostienen la legitimidad del gobierno e instituciones de Bamako. No obstante, se destaca que el hecho de que la participación en los procesos electorales de 2013 fuera tan reducida en las provincias del norte, en comparación con el sur, influye en la percepción y narrativa de algunas de las partes involucradas en el conflicto. 
influir en los distintos procesos abiertos. Concurrentemente, su dependencia económica del apoyo internacional continúa, por lo que su libertad de acción en este ámbito es, a su vez, limitada.

Durante 2012 y 2013, se iniciaron procesos relacionados con la reconciliación en forma de Comisión nacional, y se presentó el caso ante la Corte Penal Internacional (CPI). La complementariedad de elementos del proceso entre los ámbitos nacional e internacional nos permite intentar clasificar los tipos de procesos.

\section{Aproximación a la taxonomía de los procesos de reconciliación}

Con carácter general se pueden tratar de clasificar las iniciativas de consolidación de la paz en pos de la justicia y la reconciliación, en base a varias categorías:

a) Comisiones de la verdad. En las que no se depuran responsabilidades penales, el ejemplo paradigmático es la Comisión por la Verdad y la Reconciliación de Sudáfrica, establecida en $1995 .{ }^{45}$ Se optó por no incidir en la justicia retributiva. No se corresponde con el actual diseño del proceso en Malí. Es lo que Huyse califica como Comisiones de la Verdad que producen justicia histórica. ${ }^{46}$

b) Modelo nacional interno con justicia retributiva. ${ }^{47}$ Las instituciones nacionales son dueñas del proceso de esclarecimiento de la verdad, se depuran responsabilidades judicialmente y se reparan los daños. Se puede contar con el apoyo de organizaciones internacionales como las NNUU. Un ejemplo de esto puede considerarse la Comisión por la Verdad, la Justicia y la Reconciliación de Togo, establecida en $1999 .{ }^{48}$ Este modelo se aproxima al caso de estudio malí; no se puede excluir taxativamente un potencial plan para la reconciliación en Malí, que finalmente materializara los procesos judiciales en instancias nacionales exclusivamente. Sobre esto se incide posteriormente en este documento.

c) Modelos en los que se acude a instancias judiciales internacionales. A su vez, pueden darse varios tipos de casos:

- Tribunales ad-hoc establecidos por el CSNU, que prevalecen sobre los nacionales como en los casos de la exYugoslavia (ICTY) en 1993, y de Rwanda (ICTR) en $1994 .{ }^{49}$

○ Modelos híbridos o mixtos. En algunos casos de modelos mixtos, coexisten comisiones nacionales con instancias judiciales internacionales como la Corte Penal Internacional (por ejemplo, la CPI actualmente investiga hechos en Malí y en Nigeria). En Mali existe una Comisión por la Verdad, la Justicia y la Reconciliación, sus instancias judiciales nacionales cooperan con la investigación de la CPI. Incluso cabe la

45 Véase, https://www.amnesty.org/es/international-justice/issues/truth-commissions.

46 Véase, Bloomfield, Barnes, \& Huyse, op. cit., p. 97.

47 Ibid, p. 99.

48 Comisión por la Verdad, la Justicia y la Reconciliación de Togo. "A raíz de las investigaciones realizadas por la misión de investigación de las Naciones Unidas y la Comisión nacional especial de investigación independiente sobre los actos de vandalismo ocurridos antes, en el curso y después de la elección presidencial del 24 de abril de 2005 (Decreto $\mathrm{N}^{\circ}$ 2005-050/PR, de 25 de mayo de2005), las autoridades del Togo establecieron la Comisión de la Verdad, la Justicia y la Reconciliación en febrero de 2009. Esta institución tiene el mandato de arrojar luz sobre los actos de violencia de carácter político cometidos en 2005, pero también a lo largo de un período más largo (1958 a 2005), para proponer soluciones que permitan lograr la pacificación social y la reconciliación nacional." Párrafos extraídos del Comité de Derechos Humanos de NNUU, Comité de Derechos Humanos. Observaciones finales del Comité de Derechos Humanos: Togo. Documento disponible, en www2.ohchr.org/english/bodies/hrc/.../CCPR-C-TGO-CO-4-Add1_sp.doc.

49 Véase, Bloomfield, Barnes, \& Huyse, op. cit., p. 100. 
posibilidad de que la instancia judicial sea a su vez pseudo-internacional o mixta, como en Sierra Leona. ${ }^{50} \mathrm{La}$ complementariedad en la formación de tribunales, en algunos casos, puede favorecer que las sentencias sean comprendidas y aceptadas por la población. En el caso de Libia, la CPI investiga algunos hechos, y supervisa a los tribunales nacionales en el proceso contra el hijo de Gadafi, entre otros. ${ }^{52}$

- La justicia se imparte solo por instancias judiciales internacionales como la CPI.

Como antes se ha indicado, otros procesos también se relacionan con la reconciliación, como por ejemplo, las reformas del sector de la seguridad. El CSNU estipulaba recientemente que es fundamental para la lucha contra la impunidad. ${ }^{53}$ Seguridad y sistema judicial están íntimamente relacionados, ${ }^{54}$ por lo que la reforma del sector judicial debe ser parte integral de las mejoras institucionales del Estado, en el que el proceso de reconciliación pretende desarrollarse. Esto último es especialmente relevante, si la jurisdicción nacional va a estar involucrada en los procesos de justicia retributiva.

En conclusión, el proceso de reconciliación implica la superación del temor por la coexistencia no violenta, lo que demanda un nivel de seguridad física aportado por las instituciones, afecta a víctimas y perpetradores, a individuos y comunidades que se acercan empáticamente en un proceso curativo que suele implicar el esclarecimiento de la verdad, y la rendición de cuentas ante la justicia. Lo anterior supone un desarrollo institucional nacional que permita que la propiedad del proceso sea local, esfuerzo al que asisten las organizaciones internacionales. En concreto, son relevantes las reformas del sector de la seguridad (SSR) y del sector judicial, íntimamente relacionados. La complementariedad entre comisiones de reconciliación, tribunales nacionales e instancias de jurisdicción internacional, en algunos casos, puede favorecer que las sentencias sean comprendidas y aceptadas por la población.

A continuación se aborda el caso malí con más detalle, en cuestiones relacionadas con la creación de las distintas comisiones de reconciliación y los avatares del proceso político implicado, como pasos previos al estudio del caso en relación con la CPI.

\section{El proceso de diálogo, verdad, justicia y reconciliación en Malí}

El proceso de reconciliación en Malí comenzó con el gobierno de transición, durante un período en el que la prioridad era la legitimación democrática mediante procesos electorales.

50 Ibid., p. 100.

51 En Sierra Leona existe una misión de asistencia de la Comisión para la Consolidación de la Paz de las NNUU. Esta Comisión suele centrarse en países con poco desarrollo institucional, lo que no se ajusta a nuestro caso de estudio. Véase página web de "United Nations Integrated Peacebuilding Office in Sierra Leone", en http://unipsil.unmissions.org/. Cuatro de entre los seis países apoyados por la Comisión son miembros de la CEDEAO: Sierra Leona, Guinea, Guinea-Bissau, y Liberia. Véase página web de la Comisión de Consolidación de la Paz de Naciones Unidas en http://www.un.org/es/peacebuilding/index.shtml.

52 Véase página web de la Corte Penal Internacional, en

http://www.icc-

cpi.int/en_menus/icc/situations\%20and\%20cases/situations/icc0111/Pages/situation\%20index.aspx.

53 Véase, "La reforma del sector de la seguridad es vital para luchar contra la impunidad", UN (abril 2014), en http://www.un.org/spanish/News/story.asp?NewsID=29311.

54 MINUSMA aborda cuestiones de seguridad y de justicia. Véase "La comunidad internacional debe seguir apoyando la recuperación de Mali" (enero 2014), en

http://www.un.org/spanish/News/story.asp?NewsID=28496\&Kw1=minusma\#.UuybZP15N5I. La misión de NNUU, se centra en apoyar la estabilización, el proceso político y los esfuerzos de reconciliación, así como en la protección de los civiles y de los derechos humanos, por lo que necesita de efectivos en los ámbitos de la seguridad militar y policial, así como expertos en áreas civiles, entre estas la judicial. 
Fue entonces cuando se presentó el caso a la CPI, no era posible acudir a la justicia nacional. En el primer semestre de 2014, el gobierno malí redefinió el plan de acción hacia la reconciliación, sus prioridades eran el desarme y desmovilización de grupos armados en el norte, el fortalecimiento de sus instituciones de seguridad y judiciales, la presencia institucional en la totalidad del territorio, y concurrentemente la negociación de un acuerdo de paz global y definitivo. Uno de los retos singulares a los que se enfrentarán las autoridades malíes será la reintegración de ex-combatientes; de modo que cuando las acciones relacionadas con la justicia se visualicen en forma de inculpaciones y juicios, ${ }^{55}$ también tendrán que considerar el 'timing' adecuado en relación con este proceso.

\section{La percepción y la narrativa importan en el diálogo por la verdad}

En el desarrollo de la guerra, ${ }^{56}$ así como en el proceso de la resolución de un conflicto importan las audiencias extranjeras y las nacionales, y de éstas, todas las partes. La percepción de las partes importa a la hora de identificar los hechos pertinentes, tanto en el global del conflicto, como en la interpretación de los episodios de escaladas verbales. ${ }^{57} \mathrm{En}$ el juego político implicado en el diálogo hay diferentes narrativas, éstas tienen su importancia a la hora de tratar de esclarecer la verdad, tanto en las iniciativas nacionales de reconciliación impulsadas, como en las instancias judiciales (nacionales e internacionales).

En el caso de estudio, se identifica al menos la necesidad de reconciliar en tres ámbitos, en el interno de la milicia malí y de ésta con el resto de instituciones del Estado (problema esencialmente interno en el sur), el de la reconciliación norte-sur en el global del país, y el de la reconciliación local en el norte. En esta zona se dan tensiones históricas multifacéticas que explosionan en enfrentamientos étnico-tribales, que se han reproducido recientemente; por otra parte, se unen al aspecto étnico, las recientes luchas entre los terroristas yihadistas y los movimientos políticos seculares en pugna por el poder. ${ }^{59}$

A continuación se alude brevemente a lo esencial de las citadas narrativas que se oponen en Malí, y se identifican las iniciativas en pos de la reconciliación.

Para los que apoyaban el golpe militar en 2012, el conflicto se había agravado por un gobierno corrupto, que también perdió su legitimidad por inacción cómplice ante la

\footnotetext{
$55 \mathrm{La}$ doctrina política y jurídico-internacional, el uso y costumbre de las organizaciones internacionales que fomentan la implementación de una hoja de ruta con ciertas pautas, desde la crisis, hasta la estabilización y hacia la consolidación, fomenta el inicio de determinados procesos, entre éstos los judiciales.

56 El episodio de guerra interna que se vivió en Malí en 2012 afectó a varios aspectos en crisis en dicho país, se trataba de "problemas de desarrollo, emergencias políticas complejas en contextos de colapso de la economía y de los Estados, lo que supone una superación del concepto clásico tradicional de seguridad"; se trataba de una crisis más amplia, de seguridad humana globalizada que sobrepasa el ámbito regional inmediato. Véase C. De Castro Sánchez, \& C. Quesada Alcalá (2007), "El derecho internacional de la gestión de crisis". En IUGM, Realidades y Perspectivas de la Gestión Internacional de Crisis, pp. 63-64.

57 Véase Walzer, M. (2001): Guerra, política y moral, Madrid, Paidós, pp. 44-48. Michael Walzer se refería a la guerra y sobre cómo trataba de justificarse moralmente: "La realidad moral de la guerra se configura no por las acciones de los soldados, sino por las opiniones del conjunto de la humanidad" (p. 48) Señalaba la dificultad de las distintas percepciones sobre la información, que dificulta identificar los 'hechos pertinentes' para unos y otros, tanto en la política como en la guerra (p. 44).

58 Miall, H., Ramsbotham, O., y Woodhouse, T., en su guía de análisis de los conflictos incluida en Contemporary Conflict Resolution (1999), se plantean, entre otras, las siguientes preguntas: ¿Hay diferentes percepciones sobre las causas y naturaleza del conflicto entre las partes? ¿Cómo es el comportamiento actual de las partes? ¿El conflicto está en fase de escalada o desescalada?

59 Castilla Barea, "Evolución estratégica y operacional del terrorismo...", op. cit.
} 
implantación terrorista, ${ }^{60}$ la rebelión y el intento de auto-segregación del norte. Por el contrario, y por parte de sectores civiles de Bamako y de algunos militares, el golpe que depuso al Presidente Touré era ilegítimo por su propia naturaleza de fuerza, impropia de un régimen democrático. A esto se añade el hecho de una fuerte división interna en las Fuerzas Armadas de Malí. A la vez, unos y otros, acusaban a ciertas facciones de los tuaregs del norte de traición al Estado, y de una alianza con los terroristas yihadistas.

Por otra parte, muchos de entre los tuaregs del norte mantienen la percepción y la narrativa, de que ellos han sido víctimas (sentimientos en torno al concepto de "Essuf"). ${ }^{61} \mathrm{Se}$ consideran víctimas tanto del proceso de descolonización, como de los anteriores gobiernos de Bamako, algunos "represivos", incluso "racistas" contra los de piel blanca, ${ }^{62}$ todos con un insuficiente sistema judicial y poco apoyo económico al norte, y también reclaman ser víctimas recientes de los yihadistas. La narrativa desde Bamako incorpora críticas al tráfico de esclavos, y la falta de lealtad al Estado, entre otras.

\section{La primera fase, urgencia, inicio del dialogo, y elecciones}

La reconciliación entre las facciones militares (boinas verdes y rojas respectivamente) ya se impulsó por el gobierno de transición de Bamako. ${ }^{63}$ Tras los altercados de octubre de 2013 en Kati (cerca de Bamako) ${ }^{64}$, y el proceso judicial a Sanogo ${ }^{65}$ y otros mandos militares, ${ }^{66}$ el

60 Mesa García, B.: "La transformación del yihadismo en el norte de Malí: de causa política a economía crimina”, UNISCI Discussion Papers, nº 34 (enero de 2014), pp. 103-118, en

http://pendientedemigracion.ucm.es/info/unisci/UNISCI-Revista.htm.

61 Mazarrasa Rodríguez, P.: "Malí, razones profundas del conflicto en el Sahel", Instituto Español de Estudios Estratégicos (IEEE) (21 de noviembre de 2012), en www.ieee.es. En la cultura tuareg anidan conceptos como el de "Essuf", que impregnan el subconsciente cultural tuareg y alimentan un victimismo ancestral, que se alimenta con la música "Ishumar", al tiempo que la población sureña los percibe como altivos y orgullosos. Hay resentimientos culturales e históricos entre las etnias del norte y las del sur, que datan, al menos, de la época colonial.

62 En referencia a la sequía de 1973 y su éxodo parcial hacia Libia: "los tuaregs no fueron ayudados por el Gobierno de Bamako. Porque los negros son terriblemente racistas. Menosprecian a los de "piel clara", los árabes y los tuaregs. Y la ley, en Mali, no castiga el racismo.” “...los franceses han tomado Gao: el ejército francés ha dejado que la población linche a los tuaregs. Es la historia de mi pueblo: las democracias africanas nos oprimen": Meti Ag Mohamed Rhissa, entrevista, Malijet, febrero de 2013, en http://www.malijet.com/les faits_divers_au_mali/lettres ouvertes_mali/63462-interview-d\%26\%23039\%3Bunproche-d\%26\%23039\%3Biyad-ag-ghali\%3A-\%C2\%AB-c\%E2\%80\%99est-mainten.html.

63 Véase Castilla, J. C. "Reforma de las Fuerzas Armadas de Malí... ", op. cit. El 26 de junio de 2013 se escenificó una ceremonia de reconciliación y perdón entre las boinas de ambos colores, presidida por el Presidente interino y con la presencia del Presidente de la Comisión por el Diálogo y la Reconciliación (CDR), entre otras autoridades civiles y religiosas. Durante la misma, el aún capitán Sanogo pidió perdón por los incidentes pasados, supuesto fruto de la incomprensión mutua. El Presidente interino expresó su deseo de que nunca más se diera el caso de tener dos ejércitos o dos policías. Anunció la liberación inmediata de los detenidos por incidentes entre las facciones. Vídeo sobre la ceremonia, disponible en $\underline{\mathrm{http}} / / / \mathrm{www}$. panafricain.com/index.asp?page $=$ galvideos\&lang $=\mathrm{fr} \& \mathrm{pi}=17 \& \mathrm{Idmedia}=12027 \&$ idchaine $=20 \& \mathrm{cat}=0$

\section{$\underline{\& \text { tipe }=0}$}

64 El último temor ante una posible contra-reacción militar aconteció en la localidad de Kati (cercana a Bamako) entre el 30 de septiembre de 2013 y los primeros días de octubre. El Presidente IBK se vio forzado a adelantar su vuelta del viaje oficial a Francia, superada la incertidumbre inicial, dirigió un discurso público al país, en el que destacó su dureza contra ciertos militares. Véase: "Les derniers évènements au Mali au menu de la rencontre entre Hollande et IBK", RFI, octubre de 2013, en http://www.rfi.fr/afrique/20131001-mali-ibk-hollande-retourbamako-kati-soumeylou-boubeye-maiga/ y "Le président IBK en colère après son retour précipité dû aux troubles au Mali", RFI, octubre 2013, en http://www.rfi.fr/afrique/20131003-le-president-ibk-colere-apres-sonretour-precipite-lies-troubles-mali-kati-armee-mali/. Entre el 30 de septiembre y el 03 de octubre, el autor, se encontraba en Bamako, era miembro del contingente de EUTM Mali.

65 Véase selección de artículos sobre Sanogo, en http://www.rfi.fr/tag/amadou-haya-sanogo/. 
gobierno del Presidente Ibrahim Boubacar Keita (IBK) parece haber impuesto la legitimidad y superioridad de las instituciones políticas, y de las instancias judiciales nacionales, sobre el poder militar, ${ }^{67}$ respetando el proceso iniciado anteriormente. La Asociación Malí de Derechos Humanos (AMDH) se personó en el juicio a Sanogo, ${ }^{68}$ proceso no relacionado con el golpe de marzo de 2012, ${ }^{69}{ }^{70}$ sino con matanzas posteriores de boinas rojas. El proceso judicial se abrió tras los altercados citados de octubre de 2013. ${ }^{71}$

La Comisión por el Diálogo y la Reconciliación (CDR) fue anunciada por el Gobierno de transición el 06 de marzo de 2013, para reunir a todas las fuerzas políticas y sociales que respaldasen el proceso. Mohamed Salia Sokina, antiguo Ministro de Defensa en los 90, presidía la CDR. Como vicepresidentes contaba con una mujer y un hombre. Traoré Oumou Touré era la presidenta de la Coordinación de Asociaciones Femeninas de Mali (CAFO), y Meti Ag Mohamed Rhissa, era uno de los líderes del MNLA, procedente de Kidal, ex combatiente y antiguo profesor que se había reintegrado en el Cuerpo de Aduanas, y que había alcanzado el grado de Teniente Coronel. ${ }^{72}$ En abril de 2013 se nombraron a sus 30 comisionados. $^{73} \mathrm{La}$ creación de la CDR era fuertemente apoyada por la CEDEAO, su presidente había sido embajador de Malí en Burkina Faso; a su vez, el presidente burkinabé era el mediador de la CEDEAO. ${ }^{7475}$

66 Véase, "Mali: 35 Militaires Inculpés Dans Deux Affaires Liées À Une Mutinerie Et Un Charnier", Mali Actu, noviembre 2014, en

http://maliactu.net/mali-35-militaires-inculpes-dans-deux-affaires-liees-a-une-mutinerie-et-un-charnier/.

67 Como gesto vale el ejemplo del orden protocolario tras la formación del primer gobierno de IBK, tras el primer ministro, el ministro de justicia, y en tercer lugar el Ministro de Defensa y antiguos combatientes. Véase, "Le Mali se dote d'un gouvernement de réconcilation nationale" (septiembre 2013), en http://www.jeuneafrique.com/Article/ARTJAWEB20130909092141/.

68 La Asociación Malí de Derechos Humanos (AMDH) se personó en la causa. Véase,

"Mali: l'ex-capitaine Sanogo poursuivi pour «complicité d'assassinats»", RFI, 23 de abril de 2014), en http://www.rfi.fr/afrique/20140423-mali-ex-general-sanogo-poursuivi-complicite-assassinats/.

69 Véase recopilación de artículos sobre Sanogo en http://www.rfi.fr/tag/amadou-haya-sanogo/. No solo se les inculpó por los altercados de Kati de septiembre-octubre de 2013, sino por matanzas contra boinas rojas tras el contragolpe de éstos del 30 abril de 2012. Encuentro de 21 cuerpos en una fosa común.

70 Véase, "Le corps d'un officier malienne disparu en 2013 identifié par des tests ADN", Jeune Afrique, 8 de noviembre de 2014, en http://www.jeuneafrique.com/Article/DEPAFP20141108110055/mali-defense-justicearmee-malienne-mali-mali-le-corps-d-un-officier-disparu-en-2013-identifie-par-des-tests-adn.html.

71 El Informe del CSNU sobre la situación en Malí del 09 de junio de 2014, disponible en http://www.un.org/ga/search/view doc.asp?symbol=S/2014/403, cita:"Se han presentado cargos contra 21 miembros de alto rango del aparato militar y de seguridad, que han sido detenidos y están a la espera de juicio en relación con la desaparición y ejecución sumaria de 25 soldados a raíz del contragolpe de Estado de abril de 2012. El 22 de abril el General Amadou Haya Sanago y sus abogados comparecieron ante un juez para responder por los cargos de complicidad en el secuestro. Después del interrogatorio, se modificó la acusación a complicidad en el secuestro y homicidio. Sin embargo, casos menos prominentes no han avanzado."

72 Véase, "Commission dialogue et réconciliation au Mali: les premières nominations rendues officielles", RFI,

31 marzo 2013, en

http://www.rfi.fr/afrique/20130331-commission-dialogue-reconciliation-mali-premieres-nominations-renduesofficielles/.

73 Díez Alcalde, J.: "Ganar la paz para refundar Malí", Instituto Español de Estudios Estratégicos (IEEE) (13 de abril de 2013) en www.ieee.es. El autor significa el detalle del seguimiento de China de la situación en Mali, y en concreto en relación a algunos jóvenes líderes políticos. La agencia de noticias china Xinhua, relacionaba la noticia de las disputas entre tuaregs de Menaka y del MNLA con el juramento de los miembros de la recientemente constituida Comisión para el Dialogo y la Reconciliación (CDR) a finales de abril.

74 Véase, "Le Chef De La Commission Réconciliation Malienne Reçu Par Blaise Compaoré", Mali Actu, 30 de abril de 2013, en http://maliactu.net/le-chef-de-la-commission-reconciliation-malienne-recu-par-blaisecompaore/.

75 Blaise Compaoré tuvo que dejar el poder en Burkina Faso a finales de octubre de 2014, véase "Exclusif. Burkina : comment la France a exfiltré Blaise Compaoré", Jeune Afrique, 4 de noviembre de 2014, en http://www.jeuneafrique.com/Article/ARTJAWEB20141104143847/. 
En junio de 2013, aunque la CDR era apoyada por organizaciones como la Organización Internacional de la Francofonía (OIF), la desconfianza sobre el futuro de una Comisión nacida de un Gobierno de transición y en un contexto concreto era un hecho. "¿Por dónde comenzar?¿Forma parte la justicia del mandato de la Comisión? ¿Acompañarán las explicaciones al perdón? ¿Quién decidirá el perdón? ¿Cómo continuar tras las elecciones presidenciales de julio? ¿Podrá su mandato de dos años evolucionar? "Entre los que intentaban ayudar en las iniciativas de la OIF, estaba el que fue presidente de la Comisión de Verdad, Justicia y Reconciliación de Togo entre 2009 y $2012 .{ }^{76}$

La CDR iniciaba sus tareas con grandes expectativas y en un entorno muy complicado, en un momento en el que la prioridad nacional e internacional era lograr un acuerdo entre las partes, que permitiera la celebración de elecciones presidenciales a finales de julio, y del resto de comicios durante el segundo semestre de 2013, hasta conseguir la vuelta a la normalidad constitucional.

El acuerdo de Uagadugú (Burkina Faso), de 18 de junio de 2013, permitió finalmente la celebración de los comicios. En el mismo artículo 17 del acuerdo, en el que se aludía a la liberación de presos de los últimos enfrentamientos armados, se dejaba claro que, no se pararían los casos de crímenes que el Gobierno de transición había elevado a la Corte Penal Internacional (CPI) el 13 de julio de 2012. ${ }^{77}$ Asimismo se acordaba la constitución de una comisión internacional de investigación sobre estos crímenes:

“[...] las partes acuerdan que esta suspensión (de procesos en la justicia malí) no se aplique a los crímenes de guerra, a los crímenes contra la humanidad, a los crímenes de genocidio, a los crímenes de violencia sexual y violaciones graves del derecho internacional de los derechos humanos y del derecho internacional humanitario." (Acuerdo de Uagadugú, artículo 17)

En septiembre de 2013, IBK incluyó en su gobierno, el "Ministerio para la Reconciliación nacional y el Desarrollo de las regiones del norte". El nuevo ministro, Cheick Oumar Diarrah ${ }^{78}$, resaltaba el carácter intrínsecamente político del proceso de la reconciliación, y lo unía al aspecto del desarrollo de infraestructuras básicas, y la salida de la pobreza especialmente en el norte. De alguna forma se presentaba el posible incentivo económico $^{79}$ procedente de la ayuda internacional, como forma de buscar los objetivos comunes de futuro en pos de la reconciliación. En el aspecto político, se postulaba por la descentralización y no por la federalización en el norte, por no repetir los errores de la descentralización tras los acuerdos de paz (pacto nacional) de 1992, ${ }^{80} 81$ ajustando los

76 Así como un antiguo representante especial de las NNUU y mediador en Somalia entre 2007 y 2010. Véase, "Mali: un atelier pour aider la Commission dialogue et réconciliation", RFI, 9 de junio de 2013, en http://www.rfi.fr/afrique/20130609-mali-atelier-aider-commission-dialogue-reconciliation-oif/.

77 Carta presentación del caso ante la CPI, disponible en http://www.icc-cpi.int/NR/rdonlyres/A245A47FBFD1-45B6-891C-3BCB5B173F57/0/ReferralLetterMali130712.pdf.

78 Diplomático en EEUU entre 1995 y 2002. Véase selección de artículos sobre Cheick Oumar Diarrah en: http://www.jeuneafrique.com/tags/131320/cheick-oumar-diarrah.

79 Acemoglu, D., \& Robinson, J. A. (2012): Los orígenes del poder, la prosperidad y la pobreza. Por qué fracasan los países, Barcelona, Ediciones Deusto. Los autores insisten en que para que los países que han fracasado puedan llegar a la senda del desarrollo, es importante que haya instituciones políticas y de mercado inclusivas, no extractivas. Como precondición al paso de instituciones inclusivas a las extractivas, se ha de dar una incipiente capacidad estatal de proporcionar seguridad de modo centralizado. En el proceso los incentivos son importantes.

80 Keita, K. (1998): Conflict and conflict resolution in the Sahel: the tuareg insurgency in Mali, Carlisle, PA: Strategic Studies Institute, pp. 16-18.

$81 \mathrm{Si}$ en los 90, Argelia jugaba el papel de mediador entre los rebeldes tuareg y Bamako, hasta mayo de 2014, IBK parecía buscar un contrapunto en Marruecos, fomentando su papel como mediador con el MNLA y buscando su apoyo en contra de los procesos de radicalización religiosa con la formación de imanes malí acorde 
objetivos a los medios, y no repitiendo la ausencia de las fuerzas militares y policiales en el norte. También cerraba las puertas a cuestiones sobre una posible amnistía general para los crímenes de $2012 .^{82}$

\section{La segunda fase, Verdad, Justicia y Reconciliación}

En enero de 2014, el gobierno malí aprobaba por ordenanza la creación de la Comisión por la Verdad, la Justicia y la Reconciliación (CVJR); suponía el fin de la anterior CDR. El rol de la CDR en relación con las negociaciones de paz era controvertido desde el principio, y su balance general a principios de 2014 exiguo. ${ }^{83}$ El hecho de que en la denominación de la CVJR se incluya la palabra Justicia, puede implicar un posible conflicto de competencias con la CPI, a pesar del principio de complementariedad. Sobre este punto se incide posteriormente. La nueva Comisión cuenta con 15 delegados y 7 grupos de trabajo, un mandato de 3 años, y aspira a analizar el conflicto desde los años 60. Un período mayor al inicialmente planteado para la CDR (2012-2013), tal como habían reclamado representantes del MNLA. ${ }^{84}$

El 23 de enero de 2014, el CSNU se congratulaba por la celebración de las elecciones legislativas de 24 de noviembre y 15 de diciembre, pero al tiempo señalaba la fragilidad de la situación de seguridad en el norte, y reiteraba las llamadas a impulsar el proceso inclusivo de negociaciones de paz. Por otro lado, insistía dirigiéndose a los Estados sobre la necesidad de que se completaran los medios de MINUSMA para que con rapidez se completara su despliegue. Entre otros fines, para poder facilitar el acantonamiento de los grupos armados, como primera medida del proceso de DDR, parte esencial preparatoria para las negociaciones de un acuerdo global de paz pendiente. ${ }^{85}$ El CSNU hacía una llamada expresa a la responsabilidad del Estado en primera instancia (primer pilar de la responsabilidad de proteger $^{86}$ ), y al rol de NNUU:

a la corriente sufí del islam. Al respecto véase, "Au Mali, Mohamed VI veut peser sur la réconciliation nationale", Jeune Afrique, 18 de febrero de 2014, en

http://www.jeuneafrique.com/actu/20140218T183425Z20140218T183357Z/, artículo publicado con ocasión de la segunda visita del rey marroquí a Malí en menos de 6 meses.

82 "Mali: Cheick Oumar Diarrah, ministre de la Réconciliation nationale et du Développement des régions du Nord", RFI (12 septiembre 2013), en http://www.rfi.fr/emission/20130912-mali-cheick-oumar-diarrah-nordministre-reconciliation-developpement-l.

83 International Crisis Group: "Mali: Reform or Relapse", Crisis Group, Africa Report, no 10 (2004), p. 32.

84 Díez Alcalde, J.: "Presidente Keita: democracia frente a los desafíos de Mali", Instituto Español de Estudios Estratégicos (IEEE) (24 de septiembre de 2013) en www.ieee.es. El autor expone: "Ibrahim Ag Mohamed Assaleh, en nombre del MNLA, incide en la misma dirección, pero subraya que «la verdad debe llegar a todos los crímenes cometidos en el norte de Malí desde la independencia de nuestro país" e incluye la siguiente cita: "Le ministre Cheick Oumar Diarra l'a déclaré hier: «La Commission dialogue s'appellera désormais Commission vérité et réconciliation»", Mali Actu (18 septiembre 2013), en http://maliactu.net/le-ministrecheick-oumar-diarra-la-declare-hier-la-commission-dialogue-sappellera-desormais-commission-verite-etreconciliation/.

85 "Nota de prensa SC 11259", Presidential Statement S/PRST/2014/2 (23 enero 2013), en http://www.un.org/News/Press/docs/2014/sc11259.doc.htm.

86 El concepto de la "Responsabilidad de Proteger" (R2P), se relaciona en origen con el debate de la "intervención humanitaria" frente a la injerencia en la soberanía de los Estados, propio de finales del siglo pasado. Se desarrolla a partir de 2001, y no constituye una norma jurídica internacional nueva. Comenzó a gestarse a partir del documento canadiense "La responsabilidad de proteger" (International Commission on Intervention and State Sovereignty, 2001) que da forma original al concepto. En la Cumbre de 2005 de Naciones Unidas también se abordó la cuestión de la "Responsabilidad de proteger a las poblaciones del genocidio, de los crímenes de guerra, de la depuración étnica y de los crímenes de lesa humanidad". El concepto se desarrolla en informes anuales de NNUU. En 2006, el CSNU utilizó los términos R2P en una resolución genérica, la 1674 sobre los conflictos armados y la protección de civiles, mujeres y niños, reafirmando los párrafos 138 y 139 de la 
"El Consejo subraya la responsabilidad primordial del Gobierno de Malí en lo referente a la seguridad y la estabilidad en todo su territorio. El Consejo destaca la importancia de lograr sin más demoras el despliegue operacional completo de la MINUSMA para estabilizar los principales centros de población y proteger a los civiles, en particular las mujeres y los niños, especialmente en el norte de Malí, y para seguir apoyando el re-establecimiento de la autoridad del Estado en todo el país y la promoción del estado de derecho y la protección de los derechos humanos." (Declaración de la Presidencia del CSNU de 23 de enero, S/PRST/2014/2, pág 2)

Entre el 6 y el 8 de febrero de 2014, el proceso de reconciliación nacional se vio gravemente comprometido tras los choques entre peuls y tuaregs en la provincia de Gao, que causaron numerosos muertos entre la tribu tuareg de los Imrad, en la localidad de Tamkoutat; la represalia tuareg causó a su vez bajas entre los peuls. Las primeras cifras estimaban unas ochenta víctimas mortales, en abril fuentes de NNUU las cifraban en cuarenta. En noviembre de 2013, ya se habían producido choques significativos entre las mismas comunidades. Además de la historia relacionada con las milicias populares en la provincia de Gao, ${ }^{87} \mathrm{se}$ hacía de nuevo evidente que, desde que elementos de los peuls, los songhais y los árabes de la zona se integraron en MUYAO, el acceso a las armas ${ }^{88}$ era fácil, y no solo para los tuaregs. A consecuencia de lo anterior, se reactivaron las críticas a la impunidad y a la falta de justicia; las heridas del pasado, aún sin cicatrizar, se volvían a abrir. ${ }^{89} 90$

A estos enfrentamientos étnicos en el norte siguieron, manifestaciones del ministro de la reconciliación sobre el proyecto de la nueva Comisión por la Verdad, la Justicia y la Reconciliación. También anunciaba unos talleres conjuntos con MINUSMA, a realizar los

Cumbre de 2005. Se aludió directamente al concepto de R2P para justificar la intervención en Libia, pero no en Malí. La resolución 1674 se recoge expresamente en el mandato de MINUSMA (Res. 2100 de 25 abril de 2013). Se aludió directamente al concepto de R2P para justificar la intervención en Libia, pero no en Malí. Los mandatos de las misiones AFISMA (resolución 2085 de 2012) y MINUSMA en Malí no esgrimen directamente en su motivación la R2P. Sin embargo, sí se aluden indirectamente a subconceptos del R2P relacionados con las instancias o pilares de prevención y respuesta, con la protección de civiles, niños y mujeres, que se encuentran en la redacción de dichos mandatos. Son destacables las citas a las resoluciones 1612 (2005) y 1960 (2010), que permiten considerar, bajo determinadas circunstancias, que ciertos crímenes contra niños y mujeres pueden considerarse crímenes contra la humanidad y por tanto, ser susceptibles de tratarse por la Corte Penal Internacional. En los dos mandatos citados, se incluyen asimismo la obligación de contribuir a la investigación de este tipo de crímenes.

87 Véase Mazarrasa, op. cit. El autor elabora en relación a las milicias populares de los songhai y fulanis, citando grupos como "Ganda Koy ("Señores de la tierra") fundado en 1994, y otros más nuevos como Ganda Iso o el Movimiento Popular Soni Ali Ber. Ganda Koy fue disuelto en 1996 y se acusaba al ejército maliense de apoyarlo, llevó el enfrentamiento al campo étnico. En diciembre de 2011 se reactivaba. Ganda Iso ("Hijos de la Tierra").

88 Entre las actividades de NNUU, y acorde al párrafo 28 del mandato de MINUSMA (res. 2100/ 2013), se desarrolló un taller para validar un Plan de Acción Nacional 2014-2018 para la lucha contra la proliferación y tráfico ilícito de armas ligeras. Taller celebrado en Bamako entre el 12 y el 14 de febrero de 2014, organizado por el Centro para la Paz y el Desarme en África de las NNUU (UNREC) acorde a la Convención sobre "Small Arms and Light Weapons" de la CEDEAO. UNREC apoya a la Comisión de Malí sobre Armas Ligeras (Mali’s National Commission on Small Arms). Ver nota de prensa de 10 de febrero de 2014, UN, en http://www.un.org/News/Press/docs/2014/dc3477.doc.htm y "Mali: validation workshop of the 2014-2018 National Action Plan", en http://unrec.org/index/index.php?option=com_content\&view=article\&id=317:maliatelier-de-validation-dupan\&catid=66: unrec-press-releases\&lang=en\&Itemid.

89 "Mali: un leader peul plaide l'apaisement après les affrontements", RFI, 9 de febrero de 2014, en http://www.rfi.fr/afrique/20140209-mali-leader-peul-plaide-apaisement-apres-affrontements-communautairestouaregs/.

90 "Report de la mise en place de la commission dialogue, vérité et réconciliation : Un désaveu pour le ministre Diarrah", Mali Actu, 28 de febrero de 2014, en http://maliactu.info/societe/report-de-la-mise-en-place-de-la-commission-dialogue-veriteet-reconciliation-un-desaveu-pour-le-ministre-diarrah. 
días 13 y 14 de febrero, a modo de reuniones preparatorias para el proceso de paz, en el Centro Internacional de Conferencias de Bamako. Cheick Oumar Diarrah manifestaba su intención de que las discusiones y las negociaciones tuvieran lugar en el interior de Malí, y no fuera del territorio. ${ }^{91} 92$

El Proyecto de ley sobre la CVJR se aprobó en la Asamblea Nacional el 20 de marzo de 2014. Durante la votación, diputados de la oposición se abstuvieron criticando la falta de autonomía de la nueva Comisión con respecto al poder político, y la falta de participación de la sociedad civil, lo que podía minar su credibilidad. ${ }^{93}$

La CVJR tiene el mismo nombre que la Comisión que se constituyó en Togo en 2009. ${ }^{94}$ En Sudáfrica, la Comisión constituida omitía el nominativo de Justicia (CVR, en 1995). En ambos casos, eran comisiones decididas en el ámbito nacional y que rendían informes en dicho ámbito, que contaron con el apoyo de las NNUU. ${ }^{95}$

Las NNUU han prestado "apoyo a procesos judiciales de transición en más de 20 países de todo el mundo. Por ejemplo, en 2011, el apoyo a la Comisión de la Verdad, la Justicia y la Reconciliación del Togo fue decisivo para que la Comisión pudiera celebrar más de 400 audiencias. Los programas de reparación de Sierra Leona, financiados a través del Fondo para la Consolidación de la Paz, han realizado muchos actos de reparación comunitarios y han entregado beneficios parciales a 20.000 de las 32.000 víctimas registradas." 96

El informe y debate en la Asamblea Nacional de la puesta en marcha de la CVJR ya puso en evidencia algunas de las diferencias entre el gobierno (cuyo nombramiento fue

91 "Le Ministre Cheick Oumar Diarrah en conférence de presse : "«Des arrestations ont eu lieu » dans l’affaire des 30 touareg tués à
Tamkoutat", Malijet, 13 de febrero de 2014, en
http://www.malijet.com/actualte_dans_les_regions_du_mali/rebellion_au_nord_du_mali/93348-le-ministre-
cheick-oumar-diarrah-en-conf\%C3\%A9rence-de-presse-\%E2\%80\%A2-\%C2\%AB-des.html.
92 En el impulso al proceso que se da a partir de abril de 2014, sí se admite que las negociaciones se den en el
extranjero, entre otros lugares, en Tamanrasset, como en los 90. Véase, "Nord du Mali: Bamako veut relancer les
négociations", RFI, 28 de abril de 2014, en http://www.rfi.fr/afrique/20140428-nord-mali-bamako-veut-relancer-
negociations/\#./?\&_suid=1400586675797015009939174868142.
93 Véase, "Mali: la Commission vérité-justice-réconciliation prend forme", RFI, 12 de marzo de 2014, en
http://www.rfi.fr/afrique/20140312-mali-commission-verite-justice-reconciliation-met-place/ y "Vote de la loi
relative à la création de la Commission Vérité-Justice et Réconciliation à l'hémicycle: L'opposition invoque une kyrielle d'insuffisances
pour justifier son abstention", Mali Actu, 28 de marzo de 2014, en http://maliactu.info/societe/vote-de-la-loi-relative-la-
creation-de-la-commission-verite-justice-et-reconciliation-lhemicycle-lopposition-invoque-une-kyrielle-
dinsuffisances-pour-justifier-son-abs.
94 Véase, "Una delegación de la comisión de la verdad y reconciliación de togo saluda la experiencia marroquí en materia de justicia
transicional", en http://www.cndh.ma/es/bulletin-d-information/una-delegacion-de-la-comision-de-la-verdad-y-reconciliacion-de-togo-saluda-la: "La Comisión de la Verdad y Reconciliación togolés se creó el 29 de mayo de 2009 en virtud de un decreto presidencial en febrero de 2009. El comité, cuyo mandato abarca el período comprendido entre 1958-2005, tiene la misión de "preparar un informe detallado sobre la naturaleza, el alcance y las causas de los actos de violencia de carácter político cometidos en el pasado en Togo y reconstruir el contexto en el que la violencia tuvo lugar. La comisión "Verdad, Justicia y Reconciliación, que tiene un plazo de dieciocho meses, prorrogado por seis meses para llevar a cabo sus misiones y entregar al presidente de la República un informe con sus conclusiones y recomendaciones, se compone de miembros nombrados de las confesiones religiosas, organizaciones de defensa de los derechos humanos, organizaciones de mujeres, sindicatos, el colegio de abogados, el órgano del poder judicial, etc. El Presidente y el Vicepresidente y los miembros del comité son nombrados por el Consejo de Ministros".

95 Véase, "Repertorio de prácticas del CSNU sobre Comisiones y órganos de investigación", UN, en http://www.un.org/es/sc/repertoire/subsidiary_organs/commissions_and_investigations.shtml

96 Véase página web del Secretario General de NNUU sobre promoción de la justicia y el derecho internacional, en http://www.un.org/es/sg/speeches/reports/67/report-intlaw.shtml. 
anterior a las elecciones legislativas) y la cámara. ${ }^{97}$ A principios de abril de 2014, tras siete meses de gobierno, dimitió el Primer Ministro Oumar Tatam Ly, que era sustituido por uno de sus ministros, Moussa Mara. El gobierno se recomponía.

Con respecto, al proceso de diálogo y reconciliación, es significativo el hecho de que el nombre del Ministerio por la Reconciliación y el Desarrollo del norte pasara a denominarse Ministerio de Reconciliación nacional. Cheick Oumar Diarrah salió del gobierno; su labor no parece haber convencido ni a los propios en el sur, ni a los oponentes en el norte ${ }^{98}$, ni al ministerio francés de defensa. ${ }^{99} \mathrm{El}$ anteriormente ministro malí de asuntos exteriores, Zahabi Ould Sidi Mohamed, árabe de Tombuctú, antiguo ex-rebelde en los 90 del Movimiento Arabe de Azawad (MAA), se hizo cargo de la cartera de reconciliación. ${ }^{100}$ Fue uno de los protagonistas del Pacto Nacional de 1992, al que se llegó con la mediación argelina. La contribución de Marruecos a la normalización de Malí ha llegado a plantear cuestiones, de medios como Radio France Internacional (Rfi), sobre el potencial papel de mediador de Marruecos con el MNLA. ${ }^{101}$ Por su relación con el proceso de reconciliación, se destaca la continuidad inicial en sus puestos de los ministros de justicia, defensa e interior. ${ }^{102}$

Además del propio Ministro para la Reconciliación, IBK nombró a un Alto Representante del Jefe del Estado para el Dialogo inclusivo inter-malí, dedicado a impulsar el proceso. Se trata de Modibo Keita, antiguo Primer Ministro interino en 2002. ${ }^{103}$ Este pronto intentó incorporar a personalidades que tuvieron un papel central en la forja de los acuerdos de $1992,{ }^{104}$ tales como Zeidan Ag Sidalamine, quién fuera coordinador del llamado

97 Véase, "Report de la mise en place de la commission dialogue, vérité et réconciliation: Un désaveu pour le ministre Diarrah", Mali Actu, 28 de febrero de 2014, en http://maliactu.info/societe/report-de-la-mise-en-place-de-la-commission-dialogueverite-et-reconciliation-un-desaveu-pour-le-ministre-diarrah.

98 "Remaniement: Pourquoi le ministre Cheick Oumar Diarrah a été débarqué", Mali Web, 17 de abril 2014, en https://www.maliweb.net/politique/gouvernement/remaniement-ministre-cheick-oumar-diarrah-ete-debarque247162.html.

99 Véase, "Mali: un nouveau gouvernement pour relancer la réconciliation", Jeune Afrique, 12 de abril de 2014 , en http://www.jeuneafrique.com/Article/DEPAFP20140412165400/: "Le ministre français de la Défense JeanYves Le Drian a estimé, dans une récente interview à Jeune Afrique, que le processus de réconciliation nationale au Mali "n'avance pas très vite".

$100 \mathrm{El}$ anterior responsable de la diplomacia malí había conseguido estrechar lazos con Marruecos. Esto es relevante, ya que también son necesarias las relaciones con Argelia para la solución del problema en Kidal. A este respecto, es significativo que Radio France Internacional tras informar sobre los enfrentamientos en la ciudad ocurridos el 17 de mayo de 2014, y el posterior cese el fuego, ponga el foco en la visita del ministro de defensa Le Drian a Alger, especialmente en relación con la lucha contraterrorista en el norte de Malí. Véase, "Mali: cessez-le-feu respecté et otages libérés à Kidal", RFI, 20 de mayo de 2014, en http://www.rfi.fr/afrique/20140520-mali-cessez-le-feu-respecte-otages-liberes-kidalmnla/\#./?\&_suid=140058637927403696811892032933.

101 Véase, "Mohammed Benhammou, président du Centre marocain des études stratégiques", RFI, 2 de febrero de 2014, en http://www.rfi.fr/emission/20140219-cmes-mohammed-benhammou-president-maroc-etudesstrategiques/\#./?\& suid=1400586673956009350835811778368

102 Véase, "Le Mali se dote d'un gouvernement de réconcilation nationale", Jeune Afrique, 9 de septiembre de 2013, en http://www.jeuneafrique.com/Article/ARTJAWEB20130909092141/ y "Le Gouvernement du 11 avril 2014. Selon le Décret $\mathrm{N}^{\circ} 2014-0257 / \mathrm{P}-\mathrm{RM}$ du 11 avril 2014 portant nomination des membres du Gouvernement", en

http://www.primature.gov.ml/index.php?option=com_content\&view=article\&id=10337\&Itemid=100013.

103 No confundir con el que fuera Presidente de la República entre 1960 y 1968, Modibo Keita (1915-1977). El Alto Representante ocupó cargos de responsabilidad entre 2002 y 2007. Véase L'ex-Premier ministre, Modibo Keïta chargé du dialogue avec la rébellion, Malijet (23 abril 2014), en http://www.malijet.com/actualte_dans_les_regions_du_mali/rebellion_au_nord_du_mali/99888-sortie-de-crise1\%E2\%80\%99ex-pm,-modibo-ke\%C3\%AFta,-d\%C3\%A9sign\%C3\%A9-haut-repr\%C3\%A9sentant.html. 104 Junto a Modibo Keita, se incorporan a equipo por la reconciliación antiguos rebeldes como Zeïdane Ag Sidalamine. Véase, "Réconciliation nationale: Zeïdane bientôt chez Modibo Kéita", Maliweb, 15 de mayo de 
Movimiento y Frente Unificado de Azawad (MFUA). ${ }^{105}$ Modibo Keita, a finales de abril de 2014, se mostraba dispuesto a hacer concesiones para alcanzar el acuerdo definitivo de paz global, para alcanzar la reconciliación "a la luz de la justicia". ${ }^{106}$ En el Acuerdo de Uagadugú (18 junio de 2013) ya se incluía un plazo a las partes, por el que se obligaban a iniciar las conversaciones en sesenta días; plazo obviamente sobrepasado.

El 15 de abril de 2014 se entrevistaron el nuevo Primer Ministro, Moussa Mara, y el Jefe de MINUSMA, Albert Koenders. Según medios malíes, se produjo un desencuentro entre ambos acerca de las respectivas responsabilidades, a la hora de organizar y asegurar las elecciones comunales, cuya celebración entonces se preveía celebrar en el último trimestre de 2014. ${ }^{107}$ A finales de abril, RFI publicaba que la ONU demandaba el progreso en las negociaciones de paz, y poco después se apuntaba a que las conversaciones para el proceso de paz podrían desarrollarse, entre otros lugares, en la ciudad del sur de Argelia, Tamanrasset. ${ }^{108}$ A principios de mayo, se producía un reforzamiento del despliegue del contingente francés en Kidal. También se reforzaba el contingente de la MINUSMA. Esto venía a coincidir con críticas en medios de comunicación malíes sobre el mandato de la misión de la ONU, ${ }^{109}$ críticas que llegaban a cuestionar las relaciones Malí-Francia ${ }^{110}$-MINUSMA. ${ }^{111} 112$

2014, en http://www.maliweb.net/la-situation-politique-et-securitaire-au-nord/reconciliation-nationale-zeidanebientot-chez-modibo-keita-301002.html.

105 Véase, "Zeidan Ag Sidalamine, ex-coordonnateur du MFUA : « Le Pacte national est l'un des meilleurs accords de la sous-région»", Maliactu, 24 febrero 2012, en http://maliactu.net/zeidan-ag-sidalamine-excoordonnateur-du-mfua-le-pacte-national-est-lun-des-meilleurs-accords-de-la-sous-region/.

106 Véase, "Nord du Mali: l'ONU demande l'accélération des négociations", RFI, 28 de abril de 2014, en http://www.rfi.fr/afrique/20140424-nord-mali-onu-demande-acceleration-negociations-koenders-modibo-keitadiop-minusma/\#./?\&_suid=140059535298106472896433728741. «Je conçois ma mission avec l'objectif ultime d'aboutir à un accord de paix global et final ». Pour y parvenir, l'ancien Premier ministre malien, reconnu pour son intégrité, explique son état d'esprit : «Le mot 'groupe rebelle' me heurte. Ceux que je rencontre là, ce sont des Maliens, des Maliens qui ont des préoccupations. Ça suppose des concessions, ça suppose une ouverture d'esprit... » En clair, pour faire avancer les choses il faut rapidement trouver des réponses à plusieurs questions, que Modibo Kéita énumère : «Comment le Mali va-t-il organiser administrativement et institutionnellement l'ensemble du territoire ? Comment le Mali va définir un programme de développement intégré de collectivité territoriale ? Et comment doit-on s'y prendre pour améliorer la gouvernance ? Et enfin, il y a une question, la réconciliation à la lumière de la justice. Voilà des questions ! ».

107 Véase, Polémique autour de la fin de la mission de la MINUSMA ou la redéfinition de son mandat, Malijet, 7 de mayo de 2014, en

http://www.malijet.com/actualte dans_les_regions_du_mali/rebellion_au_nord_du_mali/101061-sortie-de-criseet-gestion-de-la-situation-securitaire-au-nord-d.html.

108 En la misma localidad también se forjaron acuerdos a principios de los 90. Véase, "Nord du Mali: Bamako veut relancer les négociations", RFI, 28 de abril de 2014, en http://www.rfi.fr/afrique/20140428-nord-malibamako-veut-relancer-negociations/\#./?\&_suid=1400598313855016913605700955614.

109 Véase, "Polémique autour de la fin de la mission de la MINUSMA ou la redéfinition de son mandate",

Malijet, 7 de mayo de 2014, en

http://www.malijet.com/actualte_dans_les_regions_du_mali/rebellion_au_nord_du_mali/101061-sortie-de-criseet-gestion-de-la-situation-securitaire-au-nord-d.html.

110 Con respecto a las relaciones con Francia es relevante el artículo publicado en medios malíes que recoge declaraciones del Ministro de Defensa francés y relaciona el futuro de la operación Serval con la iniciativa del G5 africano. Véase, "L'avenir de l'opération Serval au Mali", Maliweb, 8 de mayo de 2014, en http://maliweb.org/nord-mali/lavenir-de-loperation-serval-au-mali. Véase también declaraciones del Ministro francés, "Mali: «La France tient à s'installer dans la durée»", RFI, 8 de mayo de 2014, en http://www.rfi.fr/afrique/20140508-mort-soldat-francais-serval-mali-philippe-chapleau-le-drian-operationantiterrorist/\#./?\&_suid=140059894263802644213386853559

111 Véase, "Relations Mali-France-MUNUSMA: Des éclairages s'imposent!", Maliweb, 20 de mayo de 2014 , en http://www.maliweb.net/armee/relations-mali-france-munusma-eclairages-simposent-312602.html.

112 En la posterior resolución 2164 de 25 de junio, quedó claro el papel de las fuerzas de MINUSMA: "Prestar apoyo, dentro de los límites de sus recursos y sus zonas de despliegue, a la realización de elecciones locales inclusivas, libres, limpias y transparentes, entre otras cosas mediante la prestación de asistencia logística y 
El 23 de abril de 2013, el CSNU emitía una nota de prensa (SC/11360) ${ }^{113}$, en la que se destacaban ciertos aspectos del informe de situación sobre Malí (S/2014/229), ${ }^{114}$ y del proceso de reconciliación en marcha. Se señalaba la coincidencia de parecer entre el Secretario General y el Ministro malí de Asuntos Exteriores, Integración Africana y Cooperación Internacional, sobre el retraso en las negociaciones de paz motivado por la falta de cooperación de algunos grupos armados, lo que agravaba la deteriorada situación de seguridad en el norte, en particular por la reactivación de las actividades terroristas. A esto se añadía de nuevo, la constatación de la falta de capacidades de MINUSMA, para completar el nivel de fuerzas y despliegue acorde a la Resolución 2100 (2013). Como aspecto positivo se alababan las iniciativas de los talleres preparatorios de las conversaciones de paz de febrero, antes citados, y el nombramiento de un alto funcionario como representante especial del gobierno, para el proceso de reconciliación.

El 17 de mayo, durante una anunciada ${ }^{115}$ visita del Primer Ministro a Kidal, se produjeron enfrentamientos armados protagonizados por el MNLA y el ejército malí, el asalto a las oficinas del gobernador en la ciudad, la retención durante algún tiempo de funcionarios y periodistas, ${ }^{116}$ la muerte de 36 personas, entre ellos militares y funcionarios. El Primer Ministro manifestó públicamente que los acontecimientos suponían "una declaración de guerra", ${ }^{117}$ al tiempo que se reproducían críticas a la pasividad de las fuerzas de la operación Serval y de MINUSMA. ${ }^{118}$ El General Kazura, Jefe de las Fuerzas de MINUSMA, tuvo que intervenir para acordar el alto el fuego y coordinar la devolución de los prisioneros del MNLA a la Cruz Roja Internacional. El presidente IBK se dirigió a la nación por la televisión nacional. ${ }^{119}$ De su discurso, disponible en la página web oficial del gobierno, se subrayaban cuatro aspectos literales:

"El ejército malí se encontró en situación de asegurar, solo, la seguridad de los desplazamientos terrestres de la delegación del primer ministro."

técnica apropiada y arreglos de seguridad eficaces, en el contexto de un amplio proceso de descentralización con la dirección y titularidad de las autoridades de Malí". Véase,

http://www.un.org/es/comun/docs/?symbol=S/RES/2164 (2014).

113 Véase "'Window of Opportunity' for Peace in Mali Remains Open, but Requires Sustained Commitment, Senior Official Tells Security Council", UN (23 abril 2014), en

http://www.un.org/News/Press/docs//2014/sc11360.doc.htm.

114 Informe (23 abril 2014), en

http://www.securitycouncilreport.org/atf/cf/\%7B65BFCF9B-6D27-4E9C-8CD3-

CF6E4FF96FF9\%7D/s_pv_7158.pdf.

115 Véase, "Mali: visite incertaine de Moussa Mara à Kidal ce samedi", RFI, 17 de mayo de 2014, en http://www.rfi.fr/afrique/20140517-mali-visite-incertaine-moussa-mara-kidal-

samedi/\#./?\&_suid=140057719435909408144218687884.

116 Véase, "Combats à Kidal: «une trentaine de fonctionnaires portés disparus»", RFI, 18 de mayo de 2014, en http://www.rfi.fr/afrique/20140518-mali-combats-kidal-lors-visite-moussa-

mara/\#./?\& suid=1400577185468011348774230154329.

117 Véase, "Moussa Mara: «L'attaque de Kidal est une déclaration de guerre»", RFI, 19 de mayo de 2014, en http://www.rfi.fr/afrique/20140519-moussa-mara-kidal-jihadistes-mnla-rfi-cette-attaque-est-une-declarationguerre-mali/\#./?\&_suid=140057523870307333550881400366.

118 Véase, "Mali: la France a-t-elle été trop passive à Kidal?", RFI, 20 de mayo de 2014, en http://www.rfi.fr/afrique/20140519-mali-france-affrontements-kidal-accusations-moussa-mara-passiviteserval/\#./?\& suid=1400575136177020525658244558442.

119 Texto del discurso, en http://www.primature.gov.ml/index.php?option=com_content \&view=article\&id=12356:message-a-la-nation-desem-ibrahim-boubacar-keita-president-de-la-republique-chef-de-letat-suite-aux-evenements-de-

kidal\&catid=5\&Itemid=100037. Véase también, "Mali: à la télévision, IBK condamne les violences à Kidal", RFI, 20 de mayo de 2014, en http://www.rfi.fr/afrique/20140520-mali-television-ibk-condamne-violenceskidal/\#./?\&_suid=14005751215440992104731271268. 
"No permitiré que esos grupos armados, algunos calificados de terroristas, otros designados cómodamente como rebeldes, pero todos, reunidos y solidarios de nuevo, como lo hicieron cuando perpetraron, juntos, el horror de Aguelhoc, [...] no les dejaré que continúen marcando la ley, ni en Kidal, ni en ninguna otra parte de nuestro territorio."

"El gobierno de Malí desempeñará su rol plenamente. En particular, los autores de la toma de rehenes y ejecuciones sumarias serán perseguidos y llevados ante los tribunales nacionales e internacionales."

"Siendo la soberanía de Malí sobre toda la extensión de su territorio no negociable, nuestras Fuerzas de Defensa y Seguridad en reconstrucción asumirán, totalmente, las misiones que la constitución malí les asigna y que los malies esperan de ellas.”

A pesar de la contundencia del Presidente en mayo, la posición de las autoridades de Bamako con respecto a los grupos armados del norte se ha debilitado durante el segundo semestre de 2014. No hay presencia gubernamental significativa en Kidal, y la esfera de control sobre las negociaciones se ha internacionalizado. ${ }^{120}$

Tras los acontecimientos de mayo en Kidal, las iniciativas diplomáticas se focalizaron en buscar una cooperación activa de Argelia, lo que ya se introdujo anteriormente. Tras el mes de mayo de 2014, Argelia era de nuevo, como en el pasado, el escenario en el que se gestaba un posible nuevo acuerdo de paz. La resolución 2164 del CSNU de 25 de junio acogia "con satisfacción los esfuerzos de Argelia por contribuir al lanzamiento de un proceso de negociación creíble e inclusivo". 121

Una primera fase de estas negociaciones se llevaba a cabo entre los días 16 y 24 de julio en Alger; se acordaba una hoja de ruta para las negociaciones. ${ }^{122}$ A finales de septiembre se seguía negociando en Argelía, el Secretario General de NNUU ponía de manifiesto que las negociaciones se realizan con presencia de su representante y que los aspectos políticos, ${ }^{123} \mathrm{de}$ seguridad, desarrollo, justicia y reconciliación debían ser parte del acuerdo. ${ }^{124}$ Las negociaciones se desarrollan en torno a cuatro comisiones, de política, de justicia, de desarrollo y de seguridad. ${ }^{125}$ A finales de octubre se celebrara una tercera ronda de

120 Véase, documento de vídeo, "Mali: Reprise des négociations entre gouvernement et groupes armés du Nord", TV 5, 5 de septiembre de 2014, en http://www.tv5monde.com/cms/chaine-francophone/Revoir-nosemissions/Afrique-Presse/Episodes/p-29032-Mali-reprise-des-negociations-entre-gouvernement-et-groupesarmes-du-Nord.htm.

121 Véase texto de la resolución, en http://www.un.org/es/comun/docs/?symbol=S/RES/2164(2014).

122 «La phase initiale du dialogue intermalien, qui avait eu lieu du 17 au 24 juillet à Alger, avait été couronnée par la signature de deux documents comportant "la feuille de route pour les négociations dans le cadre du processus d'Alger" et une "déclaration de cessation des hostilités" entre le gouvernement du Mali et six mouvements politico-militaires du nord de ce pays». Véase, La MINUSMA appelle toutes les parties à la "retenue" suite aux récents affrontements dans le Nord, 5 noviembre 2014, en http://www.aps.dz/monde/13417-la-minusma-appelle-toutes-les-parties-\%C3\%A0-la-retenue-suite-auxr\%C3\%A9cents-affrontements-dans-le-nord.

123 El modelo de Estado, descentralizado o federal era uno de los puntos de divergencia entre las propuestas del gobierno y los grupos armados respectivamente. Véase, "Malí: à Alger, la médiation face à deux plans de sortie de crise", Jeune Afrique, 17 de octubre de 2014, en http://www.jeuneafrique.com/Article/ARTJAWEB20141017165707/mali-gouvernement-malien-mnla-crisemalienne-crise-malienne-mali-a-alger-la-mediation-face-a-deux-plans-de-sortie-de-crise.html.

124 Véase, "Secretary-General Cites Political Process in Mali as Cornerstone for Stability, Reiterates Importance of Inclusiveness, at High-Level Meeting", UN (27 de septiembre de 2014), en

http://www.un.org/press/en/2014/sgsm16219.doc.htm.

125 Véase, "Mali: négociations mode d'emploi", RFI, 5 de septiembre de 2014, en

http://www.rfi.fr/afrique/20140905-mali-negociations-mode-emploi-ramatane-lamamra-mnla-azawad-

diplomatie-algerienne/. 
negociaciones. ${ }^{126127}$ A principios de noviembre aún no se ha llegado a un acuerdo, y se hacen esfuerzos por sintonizar a los medios locales malienses en torno a un discurso favorecedor del proceso de paz. ${ }^{128}$ Está prevista reanudar las conversaciones a finales de noviembre en Argel. $^{129}$

A modo de conclusiones parciales, se significa que se ha operado un cambio sustancial en la naturaleza y mandatos de la comisión nacional por la reconciliación incluyendo el aspecto relativo a justicia. Se han incorporado reclamaciones de los tuaregs relativas al ámbito temporal sobre el que investigar la verdad en el diálogo. Se cuenta con personalidades, árabe y tuareg respectivamente, en el lado de Bamako, que tuvieron un papel significado en la elaboración de los pactos en 1992, lo que puede ayudar a no repetir errores del pasado. ${ }^{130}$ Tras la remodelación ministerial de abril se asistió a una escalada verbal por parte de instancias malíes en relación con MINUSMA principalmente. El deterioro de seguridad en el norte, se ha visto recientemente jalonado por rebrotes de enfrentamientos étnico-tribales y por los acontecimientos en Kidal de mayo, que implicaron directamente al Primer Ministro y que ha supuesto un punto de inflexión en relación con el proceso de reconciliación, cuyo futuro está marcado de nuevo por la influencia mediadora de Argelia. La prioridad se centra en seguir impulsando el proceso de paz. Las elecciones locales que deberían celebrarse en el último trimestre del año son aún una incógnita. Las intensas negociaciones en Argelia, entre julio y octubre, buscan alcanzar el acuerdo de paz definitivo, pendiente desde el acuerdo preliminar de Uagadugú de junio de 2013.

Un día antes de los últimos enfrentamientos armados en Kidal, el 16 de mayo, se presentaba una encuesta del Afrobarómetro, ${ }^{131}$ realizada en diciembre de 2013, sobre el proceso de reconciliación nacional. ${ }^{132}$ Según la encuesta, el $64 \%$ de los malíes se decantaba por la punición de crímenes. El 90\% percibía que el principal rol de la CVJR era el de llevar a los culpables ante los tribunales, mientras que el $79 \%$ pensaba que debía promover el diálogo intercomunitario. El $47 \%$ de los malíes se postulaba a favor de llevar a los culpables ante los tribunales nacionales, mientras que el $25 \%$ prefería la jurisdicción internacional.

A finales de octubre, el Primer Ministro malí, Moussa Mara afirmaba que la justicia no podía ir más rápido que la seguridad en el norte, y que había crímenes que no prescribían. ${ }^{133}$ A principios de noviembre se celebró un seminario internacional en Bamako titulado "La

126 Véase, "Mali: réouverture des negotiations de paix à Alger", RFI, 20 de octubre de 2014, en http://www.jeuneafrique.com/Article/ARTJAWEB20141020090643/.

127 El presidente francés urgía a IBK en la dirección de llegar a un acuerdo político, una vez que estaba demostrado que la solución militar no era posible. Véase, "Alger: 3e partie des négociations pour le dialogue inter-malien", RFI, 19 de octubre de 2014, en http://www.rfi.fr/afrique/20141019-conflit-mali-ouverture-3epartie-negociations-alger-dialogue-inter-malien-azawad/.

128 Entre los días 5 y 7 de noviembre se celebró en Bamako el seminario, « Médias et processus de paix au Mali» (Los medios y el proceso de paz malí), Unión Africana (7 noviembre 2014), en http://www.peaceau.org/fr/article/cloture-du-seminaire-medias-et-processus-de-paix-au-mali.

129 La última actualización de este artículo se realizó el 08 de noviembre de 2014.

$130 \mathrm{Al}$ Moustapha Touré, M. (2012): What is the extent of Al Qaeda in the Islamic Maghreb and where it derive its strength in the Sahelian-Saharan Region: A case study of Northen Mali, Command and General Staff College, Fort Leavenworth, KS, EEUU.

131 Bratton, M.; Coulibaly, M., \& Dulani, B.: "Malians Want a United Country, Post-Conflict Justice Afrobarometer", Afrobarómetro, Policy Paper, nº 13 (16 mayo 2014), en

http://www.afrobarometer.org/files/documents/policy brief/ab r5 5 policypaperno13.pdf

132 Véase, "Réconciliation Nationale au Mali : La justice avant tout, préfèrent 92\% des Maliens!", Maliweb, 20 mayo 2014, en

http://www.maliweb.net/societe/reconciliation-nationale-au-mali-justice-preferent-92-maliens-312752.html.

133 Véase, "Mali - Moussa Mara- «La justice a de la mémoire»", Jeune Afrique, 31 de octubre de 2014, en http://www.jeuneafrique.com/Article/JA2806p039.xml0/corruption-moussa-mara-armee-malienne-

gouvernement-malien-mali-mali-moussa-mara-la-justice-a-de-la-memoire.html. 
reconciliación nacional. El lugar de la justicia transicional" que contaba con la presencia de representantes de la Federación Internacional de los Derechos Humanos (FIDH) y de su filial malí (AMDH), así como con autoridades gubernamentales. ${ }^{134}$ El momento y la forma de la justicia dentro del proceso de reconciliación están aún por definir.

\section{Malí en la Corte Penal Internacional. La complementariedad}

Se conoce que la Corte Penal Internacional (CPI) actúa en complementariedad con las instancias judiciales nacionales. En este apartado del trabajo se razona que técnicamente es posible que las autoridades políticas malienses decidan qué individuos se juzgan por los tribunales nacionales y cuales por la CPI.

Los factores que orienten las correspondientes decisiones son tanto de carácter endógeno como exógenos. Por un lado la población local ha de comprender el porqué de acudir a una u otra instancia, lo que ayudará a la aceptación de las sentencias. Por otra parte, el gobierno actual cuenta con mayor legitimidad que el gobierno de transición que presentó el caso a la Corte, y por tanto, se encuentra en mejor disposición de influir con decisiones políticas. No obstante, es deseable que aquellas que puedan afectar a la evolución del caso en dicha instancia, cuenten con la aceptación internacional mayoritaria. De lo contrario, las autoridades locales se arriesgan a costes para la credibilidad estatal ante otros países. Igualmente, decisiones políticas controvertidas pueden ser aprovechadas en el futuro por las partes beligerantes en el conflicto, lo que en sí, sería un elemento más de fragilidad para la reconciliación definitiva.

En este bloque se recuerdan aspectos pertinentes de la apertura del caso, se trata con mayor detalle la cuestión de la jurisdicción en aras a comprender la complementariedad de instancias. Finalmente se revisan algunas cláusulas del Tratado de Roma, por el que se rigen los signatarios de la CPI, para explorar algunos aspectos sobre la posible reversibilidad, o aplicabilidad limitada de los juicios en esta instancia.

\section{Presentación de parte e inicio de la investigación}

El 16 de enero de 2013, la CPI aceptaba investigar el caso presentado por el gobierno de transición malí, con fecha de 13 de julio. Se pedía investigar los crímenes acontecidos desde enero de 2012, en virtud del artículo 14 del Tratado de Roma, ${ }^{135}$ y en virtud de que entonces el sistema local era incapaz de perseguir y juzgar a los culpables. ${ }^{136}{ }^{137}$ Malí era el quinto país africano que aceptaba o se dirigía a la jurisdicción de la CPI en su territorio. ${ }^{138}$

134 Véase, "Réconciliation nationale La place de la justice transitionnelle" (6 noviembre 2014), en http://www.primature.gov.ml/index.php?option=com_content $\&$ view=article \&id=12896:reconciliation-nationalela-place-de-la-justice-transitionnelle-\&catid=5\&Itemid=100037.

135 Texto íntegro disponible, en http://www.boe.es/boe/dias/2002/05/27/pdfs/A18824-18860.pdf.

136 Véase, ICC Prosecutor opens investigation into war crimes in Mali: "The legal requirements have been met. We will investigate", Corte Penal Internacional (16 enero 2013), en http://www.icccpi.int/en menus/icc/press\%20and\%20media/press\%20releases/news\%20and\%20highlights/Pages/pr869.aspx.

137 Carta de referencia del gobierno malí, en http://www.icc-cpi.int/NR/rdonlyres/A245A47F-BFD1-45B6891C-3BCB5B173F57/0/ReferralLetterMali130712.pdf.

138 Véase, "Mali becomes fifth African state to either refer or accept icc jurisdiction for crimes committed on its own territory", International Justice Project, 15 febrero 2013, en

http://www.internationaljusticeproject.com/mali-becomes-fifth-african-state-to-either-refer-or-accept-iccjurisdiction-for-crimes-committed-on-its-own-territory/. 
Desde entonces, NNUU ha venido recordando la obligatoriedad de que todos los actores involucrados colaboren en las investigaciones:

"El Consejo de Seguridad recuerda que se debe hacer rendir cuentas a los responsables de violaciones del derecho internacional humanitario y de violaciones y abusos de los derechos humanos y, en este sentido, alienta al Gobierno de Malí a realizar investigaciones sobre dichos actos y a llevar a los responsables ante la justicia sin demoras, así como a seguir cooperando con la Corte Penal Internacional." (Declaración de la Presidencia del CSNU de 23 de enero, S/PRST/2014/2, pág 3)

La CPI rinde informes periódicos de sus actuaciones a las NNUU. En el informe anual que cubría el periodo entre el 1 de agosto de 2012 y el 31 de julio de 2013 , se incluía lo siguiente: ${ }^{139}$

"Desde el comienzo de la investigación, el equipo ha llevado a cabo 12 misiones de investigación en 4 países." (párr. 82 del informe) [...]Entre otras cosas, la Fiscalía presta atención particular a las acusaciones relativas a los ataques selectivos dirigidos contra edificios dedicados a la religión y los monumentos históricos, de conformidad con el artículo 8 2) e) iv) del Estatuto de Roma, incluidos los que figuran en la lista del Patrimonio Mundial, y en tal sentido ha cooperado con la Organización de las Naciones Unidas para la Educación, la Ciencia y la Cultura (UNESCO). La Fiscalía también ha solicitado la cooperación de varios otros organismos de las Naciones Unidas presentes en Malí, incluida la Misión Multidimensional Integrada de Estabilización de las Naciones Unidas en Malí (MINUSMA)."(párr. 84 del informe)

Por otra parte, la Fiscalía firmó un acuerdo de cooperación judicial con el Gobierno de Malí en febrero de 2013 (párr. 107 del informe).

En el informe a la asamblea de signatarios de la CPI, documento ICC SP/12/42 de finales de 14 de octubre de $2013,{ }^{140}$ se informaba de que la CPI estaba en negociaciones para la firma de un Memorando de Entendimiento (MOU) con MINUSMA, y se manifestaba el apoyo que venía recibiendo de la misión (párr.21) Asimismo, la CPI se congratulaba de la propuesta malí de inclusión de un párrafo en el mandato de MINUSMA (resolución 2100) que autorizara operaciones de fuerza en apoyo, tanto de las autoridades nacionales como en apoyo de la CPI (párr. 46 del informe). ${ }^{141}$ Se trata del artículo 16, g):

g) Apoyo a la justicia nacional e internacional.

Prestar apoyo, cuando sea viable y apropiado, a los esfuerzos que realicen las autoridades de transición de Malí, sin perjuicio de sus responsabilidades, para detener y llevar ante la justicia a los responsables de los crímenes de guerra y crímenes de lesa humanidad cometidos en Malí, teniendo en cuenta la remisión por las autoridades de transición de Malí de la situación imperante en su país desde enero de 2012 a la Corte Penal Internacional.

La investigación abierta en el seno de la CPI sobre el caso de Malí estuvo presente durante la elaboración del mandato de MINUSMA, y contó con la total colaboración del

139 Informe de la CPI de 13 de agosto de 2013, en

http://www.icc-

cpi.int/en menus/icc/reports\%20on\%20activities/court\%20reports\%20and\%20statements/Documents/9threport/N1342656.pdf.

140 Informe disponible, en http://www.icc-cpi.int/iccdocs/asp docs/ASP12/ICC-ASP-12-42-ENG.pdf.

141 En alusión a: See page 8 of the resolution: "To support, as feasible and appropriate, the efforts of the transitional authorities of Mali, without prejudice to their responsibilities, to bring to justice those responsible for war crimes and crimes against humanity in Mali, taking into account the referral by the transitional authorities of Mali of the situation in their country since January 2012 to the International Criminal Court". 
gobierno de transición. La colaboración se ha mantenido con los gobiernos posteriores y con MINUSMA. ${ }^{142}$

\section{La cuestión de la jurisdicción. Concurrencia y complementariedad}

Por otro lado, la complementariedad implica dilucidar sobre posibles conflictos de competencia entre las instancias judiciales malíes y la CPI. La Comisión (CVJR) incluye la palabra Justicia en su denominación, entre otros aspectos, se pretende investigar los crímenes contra las violaciones graves de los Derechos Humanos cometidos contra las mujeres, y los niños, contra los casos de atentados a la memoria individual y colectiva, y contra el patrimonio cultural, como la destrucción de los mausoleos en Tombuctú y otros, y tomar medidas de reparación, en esencia el mismo tipo de crímenes presentados a la Corte. ${ }^{143}$

Es un principio general del Derecho Penal el que nadie puede ser juzgado en más de una ocasión por los mismos hechos, sea ante instancias distintas o ante la misma (non bis in ídem). ${ }^{144}$ Se plantea la cuestión sobre la posible concurrencia, o conflicto de competencia, entre un proceso nacional basado en el principio de territorialidad (locus delicti), y el seguido por la CPI, ${ }^{145}$ a pesar del carácter complementario de esta instancia internacional con respecto al sistema judicial nacional.

El informe del fiscal de la CPI de 16 de enero de 2013, ${ }^{146}$ recoge los aspectos de jurisdicción aplicables al caso:

a) En razón del tiempo (Jurisdiction ratione temporis). Malí es signatario del Estatuto de Roma desde el 17 de julio de 1998, y lo ratificó el 16 de agosto de 2000, se entiende que la jurisdicción es aplicable a partir de 2002 en adelante, ante casos de crímenes contra la humanidad, crímenes de guerra, y genocidio. Dado que las autoridades estatales malíes enviaron el caso refiriéndose a los crímenes cometidos "desde enero de 2012" y sin fecha límite final, entiende que se pueden investigar todos los que ocurran desde entonces en el contexto malí.

b) En razón del territorio o la persona (Jurisdiction ratione loci/jurisdiction ratione personae). La jurisdicción de la CPI se extiende a la totalidad del territorio malí y a todos sus nacionales.

142 En el último informe anual, de 18 de septiembre de 2014, se especifica que se estaba finalizando un memorando de entendimiento para formalizar la cooperación entre MINUSMA y la CPI en Malí. Véase, el informe en http://www.icc-cpi.int/iccdocs/presidency/ICC-Rep-UNGA-30-10-2014-Spa.pdf

143 Véase, "Commission Vérité, Justice et Réconciliation, mode d'emploi", Mali Actu, 6 marzo 2014, en http://maliactu.info/societe/commission-verite-justice-et-reconciliation-mode-demploi

144 Véase, Rodríguez Yagüe: "La justicia universal y el principio de ne bis in ídem”, Instituto de derecho penal europeo e internacional, disponible en

http://portal.uclm.es/descargas/idp_docs/doctrinas/justicia\%20universal\%20y\%20principio\%20ne\%20bis\%20in \%20idem.pdf. Rodriguez Yagüe cita a Pigrau Solé, A.: «Hacia un sistema de justicia internacional penal: cuestiones todavía abiertas tras la adopción del Estatuto de Roma de la Corte Penal Internacional», Creación de una jurisdicción penal internacional", Colección Escuela Diplomática, no 4 (2000), pp. 70 y 71 . En cuanto a la relación entre ambas, señala este autor que podría llegar a darse el supuesto de que la Corte Internacional de Justicia, en tanto tiene competencia para la interpretación de los tratados internacionales que contienen tipos penales, considerara la inexistencia de un genocidio y la falta de responsabilidad internacional del Estado, mientras que la CPI apreciara la existencia del mismo y juzgara a personas por su comisión.

145 Véase Collantes, Jorge L. (2002): "El impacto del Estatuto de Roma en la jurisdicción sobre crímenes internacionales", Revista Electrónica de Ciencia Penal y Criminología (RECPC 04-07), en $\underline{\text { http://criminet.ugr.es/recpc/recpc 04-07.pdf }}$

146 "Situation in Mali", Article 53(1) Report (16 enero 2013), en http://www.icccpi.int/en_menus/icc/situations\%20and\%20cases/situations/icc0112/Documents/SASMaliArticle53_1PublicRep ortENG16Jan2013.pdf\#search=Article\%2053\%281\%29\%20Report\%20by\%20the\%20Office\%20of\%20the\%20 Prosecutor. 
c) En razón a la materia (Jurisdiction ratione materiae). Trata sobre asesinatos, ejecuciones sin procesos, mutilaciones tratos crueles y torturas, ataques intencionados contra objetos protegidos, pillajes y violaciones; todos ellos con la consideración de crímenes de guerra (artículo 8 del Tratado). No se consideraban crímenes de lesa humanidad en ese momento (artículo 7 del Tratado).

Sobre la complementariedad de la actuación de la CPI, el fiscal afirmaba que en enero de 2013, no había posibilidad de juzgar en Malí a los posibles culpables, ni había juicios abiertos en otros países (párr. 9). Además se señalaba que en virtud del artículo 53 (1)(b) (del Tratado de Roma) los casos potenciales que pudieran aparecer serían admisibles, es decir, "La causa es o sería admisible de conformidad con el artículo 17" (cuestiones de admisibilidad).

\section{Algunas claves en virtud del Tratado de Roma}

A continuación se plantean algunas preguntas extremas dado el contexto actual, pero pertinentes. ¿Es posible que una vez abierta la investigación del fiscal se pueda suspender el caso? La suspensión por periodos de 12 meses cabe según el artículo 16, con resolución previa del CSNU. ${ }^{147}$ ¿Cabría impugnar la competencia de la CPI, por parte de las autoridades malíes? El artículo 19.2.b alude a la posibilidad de que se impugne la admisibilidad de la causa, "Un Estado que tenga jurisdicción en la causa porque está investigándola o enjuiciándola o lo ha hecho antes". Pero en este caso, la admisibilidad ya se determinó y no fue impugnada, bien al contrario, fue a instancia de parte malí. Pero el artículo 19.4 abre la posibilidad de que un Estado impugne, antes de la celebración del juicio de la CPI, en base a que (artículo 17.1.c) "la persona de que se trate haya sido ya enjuiciada por la conducta a que se refiere la denuncia, y la Corte no pueda adelantar el juicio con arreglo a lo dispuesto en el párrafo 3 del artículo 20".

Por todo lo anterior, cabe concluir que en el caso extremo de que, las autoridades malíes consideraran adecuado que un determinado individuo se juzgara por la jurisdicción nacional, sería posible hacerlo, adelantando en el tiempo el juicio, con respecto al que potencialmente pudiera celebrarse en la CPI; esto podría ser coherente con los llamamientos del CSNU, "llevar a los responsables ante la justicia sin demoras". Es de notar, que posiblemente tendría un coste político internacional a valorar, caso de que no se hiciera con la aquiescencia internacional, y de la propia CPI, lo que requeriría de una coordinación diplomática previa.

En suma, se estima que decidir qué casos se juzgarán mediante la justicia nacional y cuáles mediante la CPI, es una cuestión esencialmente política; la decisión soberana de las autoridades legítimas puede ser decisiva. Caso de que se opte en algunos casos por la justicia nacional, se habrá también cumplido el fin instrumental de la CPI de promover la justicia en dichas instancias.

\section{Conclusiones}

El proceso de reconciliación implica la superación del temor por la coexistencia no violenta, lo que demanda un nivel de seguridad física aportado por las instituciones, afecta a víctimas y

147 Tratado de Roma, artículo 16. "En caso de que el Consejo de Seguridad, de conformidad con una resolución aprobada con arreglo a lo dispuesto en el Capítulo VII de la Carta de las Naciones Unidas, pida a la Corte que no inicie o que suspenda por un plazo de doce meses la investigación o el enjuiciamiento que haya iniciado, la Corte procederá a esa suspensión; la petición podrá ser renovada por el Consejo de Seguridad en las mismas condiciones." 
perpetradores, a individuos y comunidades, que se acercan empáticamente en un proceso curativo que suele implicar el esclarecimiento de la verdad, y la rendición de cuentas ante la justicia. Lo anterior supone un desarrollo institucional nacional que permita que la propiedad del proceso sea local, esfuerzo al que asisten las organizaciones internacionales. En concreto, son relevantes las reformas del sector de la seguridad (SSR) y del sector judicial, íntimamente relacionados. La complementariedad entre comisiones de reconciliación, tribunales nacionales e instancias de jurisdicción internacional, en algunos casos, puede favorecer que las sentencias sean comprendidas y aceptadas por la población.

El proceso de reconciliación en Malí comenzó con el Gobierno de transición, durante un tiempo en el que la prioridad era la legitimación democrática de un nuevo Gobierno, y posteriormente de una Asamblea Nacional mediante procesos electorales.

Hasta ahora, cabe distinguir dos fases en el proceso de reconciliación malí, marcadas por la vuelta a la normalidad constitucional tras los procesos electorales que culminaron a finales de 2013. La primera iniciada por el Gobierno de transición el proceso, se configuraba en base a una comisión nacional tendente al esclarecimiento de la verdad, en conjunción con la apelación a la Corte Penal Internacional, para la investigación de los crímenes de la última crisis ocurrida desde principios de 2012. La segunda comienza a materializarse con la sustitución de la Comisión del Diálogo y la Reconciliación (CDR) por la Comisión por la Verdad, la Justicia y la Reconciliación (CVJR) a principios de 2014.

Este cambio supone aumentar el período analizado a toda la extensión del conflicto histórico desde la descolonización, e incluir las instancias judiciales nacionales en la posible investigación y procesos judiciales que correspondan. La complementariedad con la jurisdicción de la Corte Penal Internacional es una clave que se mantiene por ahora, en cuanto a la investigación de crímenes cometidos a partir de 2012.

La elección sobre los individuos que serán juzgados por una u otras instancia judicial es aún una decisión por tomar, en la que influirán muchos factores, entre otros, los políticos del momento, y los históricos. Estos y los factores culturales pueden también a ayudar a que las sentencias en el futuro sean comprendidas y aceptadas.

De la primera fase se significan cuatro aspectos. Primero, la presentación por parte del Estado malí de su caso ante la Corte Penal Internacional (CPI), que ocasiona la apertura de la correspondiente investigación a partir de enero de 2013. En segundo lugar, el inicio de la reconciliación en el ámbito de las Fuerzas Armadas locales. También parece destacable el exiguo resultado de la CDR. Finalmente, es reseñable que el acuerdo preliminar de Uagadugú, de 18 junio de 2013, que postulaba un plazo de dos meses para el inicio de las negociaciones de paz definitivas, fue fundamental para permitir la celebración de las primeras elecciones tras el estallido de la crisis. La vuelta a la normalidad constitucional se completaba tras cuatro procesos electorales en 2013 (dos vueltas presidenciales y dos legislativas respectivamente).

De la segunda fase se destaca que IBK afrontó episodios difíciles. Episodios disonantes de militares, a principios de octubre de 2013, que se acallaron finalmente con el proceso a Sanogo, en instancias judiciales nacionales. Se afrontan la continuidad de la actividad terroristas y rebrotes de violencia étnico-tribal. La CDR fue reemplazada por la CVJR, aumentando el período objeto de esclarecimiento e incluyendo en el proceso al sistema judicial nacional. Se solventó una crisis gubernamental con la consiguiente remodelación en abril de 2014. Concurrentemente se detectaba una corriente soberanista que trataba de reconfigurar las relaciones de Malí en el ámbito internacional, en relación al menos con el proceso de reconciliación. El nuevo gobierno impulsaba decididamente las iniciativas relacionadas, por un lado, con la presencia institucional en el norte, y por otro, con el posible comienzo de las negociaciones de paz. 
Los graves enfrentamientos ocurridos en Kidal el 17 de mayo, con ocasión de la visita del Primer Ministro, marcó un punto álgido en las críticas locales hacia la supuesta pasividad de las tropas internacionales. Además supuso una escalada verbal por parte del Primer Ministro, y de la propia Presidencia de la República. IBK, en un discurso televisado, enfatizó la soledad de sus fuerzas militares frente a los grupos armados rebeldes o terroristas, que de nuevo actuaron juntos como en los crímenes del pasado. Asimismo, y dada su responsabilidad, mostró su determinación a que se cumpliera la ley en Kidal, y a que se llevara a los culpables ante los tribunales nacionales e internacionales. Además resaltó que la soberanía malí y su integridad territorial no eran negociables, y su determinación a que las Fuerzas Armadas, en reconstrucción, asumieran totalmente sus misiones legalmente establecidas.

Lo anterior puso el proceso de reconciliación ante un momento de expectación en relación a varios aspectos relevantes: la situación en Kidal, la posible afectación a la evolución de las negociaciones, las relaciones entre las instituciones gubernamentales y las organizaciones multinacionales en el territorio en general y en el terreno en particular, y la posible complementariedad entre los procesos que se decidan llevar ante la justicia nacional y la internacional. Los acontecimientos en Kidal supusieron la inflexión definitiva hacia un nuevo marco de mediación argelina para las negociaciones de paz. Se han mantenido rondas de diálogo entre julio y octubre; el inconcluso proceso ha de continuar con una tercera ronda de negociaciones en el mes de noviembre.

Se considera que la situación en Kidal seguirá marcando el futuro inmediato del acantonamiento, desarme y desmovilización de grupos armados no terroristas. Esta evolución será significativa para la buena marcha de las negociaciones de paz. Por otra parte, la situación en las provincias norteñas, Kidal en particular, influirá en el posible desarrollo de los comicios locales anteriormente previstos para el segundo semestre de 2014. Por último, cabe plantearse que la evolución política interna y su relación con los ámbitos internacionales, tengan su reflejo en las decisiones futuras sobre la complementariedad de los ámbitos judiciales nacionales y de la Corte Penal Internacional. Técnicamente cabe admitir que las autoridades malíes pueden impulsar el que determinados criminales sean juzgados por sus propios tribunales, y otros los sean por la CPI. Los factores que orienten las correspondientes decisiones son tanto de carácter endógeno como exógenos.

Conviene asegurarse de que la población comprenda y acepte las sentencias en el futuro. Se ha de comprender el porqué de acudir a una u otra instancia judicial, lo que ayudará a la aceptación de las sentencias. Es de esperar que el Gobierno de Bamako consensue previamente, por las vías diplomáticas, las decisiones políticas que vaya a tomar al respecto. De lo contrario, las autoridades locales se arriesgan a costes para la credibilidad estatal ante otros países. Igualmente, decisiones políticas controvertidas pueden ser aprovechadas en el futuro por las partes beligerantes en el conflicto, lo que en sí, sería un elemento más de fragilidad para la reconciliación definitiva.

Otro de los retos a los que posiblemente se enfrentarán en el futuro las autoridades será la reintegración de ex-combatientes; de modo que convendría que cuando las acciones relacionadas con la justicia, se hagan visibles, el desarme y la desmovilización de facciones armadas en el norte se haya completado con anterioridad. 